\title{
The Role of Genetic Variation of BMI, Body Composition, and Fat Distribution for Mental Traits and Disorders: A Look-Up and Mendelian Randomization Study
}

\author{
Triinu Peters ${ }^{1 *}$, Lena Nüllig ${ }^{1}$, Jochen Antel ${ }^{1}$, Roaa Naaresh ${ }^{1}$, Björn-Hergen Laabs ${ }^{2}$, \\ Lisa Tegeler ${ }^{1}$, Chaima Amhaouach ${ }^{1}$, Lars Libuda', Anke Hinney ${ }^{1}$ and \\ Johannes Hebebrand ${ }^{1}$
}

${ }^{1}$ Department of Child and Adolescent Psychiatry, Psychosomatics and Psychotherapy, University Hospital Essen, University of Duisburg-Essen, Essen, Germany, ${ }^{2}$ Institute of Medical Biometry and Statistics, University of Lübeck, University Hospital Schleswig-Holstein, Lübeck, Germany

OPEN ACCESS

Edited by:

Dimitrios Avramopoulos, Johns Hopkins University,

United States

Reviewed by:

Donald Lyall,

University of Glasgow,

United Kingdom

Peristera Paschou,

Purdue University, United States

*Correspondence:

Triinu Peters

triinu.peters@uni-due.de

Specialty section:

This article was submitted to Behavioral and Psychiatric Genetics,

a section of the journal

Frontiers in Genetics

Received: 26 August 2019 Accepted: 26 March 2020

Published: 21 April 2020

Citation:

Peters T, Nüllig L, Antel J, Naaresh R, Laabs B-H, Tegeler L, Amhaouach C, Libuda L, Hinney A and Hebebrand J (2020) The Role of Genetic Variation of BMI, Body Composition, and Fat Distribution for Mental Traits and Disorders:

A Look-Up and Mendelian

Randomization Study.

Front. Genet. 11:373.

doi: 10.3389/fgene.2020.00373
Anthropometric traits and mental disorders or traits are known to be associated clinically and to show genetic overlap. We aimed to identify genetic variants with relevance for mental disorders/traits and either (i) body mass index (or obesity), (ii) body composition, (and/or) (iii) body fat distribution. We performed a look-up analysis of 1,005 genomewide significant SNPs for BMI, body composition, and body fat distribution in 15 mental disorders/traits. We identified 40 independent loci with one or more SNPs fulfilling our threshold significance criterion $\left(P<4.98 \times 10^{-5}\right)$ for the mental phenotypes. The majority of loci was associated with schizophrenia, educational attainment, and/or intelligence. Fewer associations were found for bipolar disorder, neuroticism, attention deficit/hyperactivity disorder, major depressive disorder, depressive symptoms, and well-being. Unique associations with measures of body fat distribution adjusted for BMI were identified at five loci only. To investigate the potential causality between body fat distribution and schizophrenia, we performed two-sample Mendelian randomization analyses. We found no causal effect of body fat distribution on schizophrenia and vice versa. In conclusion, we identified 40 loci which may contribute to genetic overlaps between mental disorders/traits and BMI and/or shape related phenotypes. The majority of loci identified for body composition overlapped with BMl loci, thus suggesting pleiotropic effects.

Keywords: body composition, abdominal obesity, body mass index, mental disorders, mental traits, genetic overlap, GWAS, Mendelian randomization

\section{INTRODUCTION}

Genome-wide association studies (GWAS) have recently identified a large number of genome-wide significant loci with an impact on body mass index (BMI; $\left.\mathrm{kg} / \mathrm{m}^{2}\right) /$ obesity, body composition, and body fat distribution on the one hand, and independently on mental disorders and quantitative mental traits on the other hand. The most recent GWAS for anthropometric traits were all published in 2019 substantiating the rapidly evolving dissection of their polygenic basis: (a) for BMI, 941 near-independent genome-wide significant SNPs at 536 polygenic loci have been detected 
(Yengo et al., 2018). (b) A GWAS for whole-body lean body mass (LBM; adjusted for sex, age, and height with or without fat mass adjustments) revealed seven LBM loci (Karasik et al., 2019). Another very recent GWAS for the proportion of body fat distribution in arms, legs, and trunk on 362,499 individuals from the UK Biobank identified 98 independent loci, 29 of which had not previously been associated with anthropometric traits including BMI (Rask-Andersen et al., 2019). (c) The most recent GWAS for body fat distribution as measured by waist-to-hip ratio adjusted for BMI ( $\mathrm{WHR}_{\text {adjBMI }}$ ) reported 463 independent signals $\left(P<5 \times 10^{-9}\right)$ in 346 loci (Pulit et al., 2019). Because BMI and waist circumference (WC), hip circumference (HC), and WHR are more or less strongly correlated in adults (correlation coefficients of approximately 0.8 for BMI,WC, and $\mathrm{HC}$, respectively; 0.3 for BMI and WHR; see e.g., Schneider, 2010), WC, HC, and WHR have been analyzed as such in GWAS (Heard-Costa et al., 2009; Wen et al., 2014; Shungin, 2015), but have also been adjusted for BMI (Shungin, 2015; Graff et al., 2017; Pulit et al., 2019).

Substantial progress has also been achieved toward the elucidation of the polygenic basis of several mental disorders [schizophrenia (Ripke, 2014), autism (ASD; Grove et al., 2017), major depressive disorder (MDD; Wray et al., 2018), bipolar disorder (BD; Stahl et al., 2017), attention deficit/hyperactivity disorder (ADHD; Li et al., 2014; Demontis et al., 2018), anxiety disorders (Otowa et al., 2016), and anorexia nervosa (AN; Duncan et al., 2017)] and quantitative mental traits [intelligence (Sniekers et al., 2017; Hill et al., 2018), educational attainment (Okbay, 2016b; Lee et al., 2018), tiredness (Deary, 2018), openness (de Moor et al., 2012), conscientiousness (de Moor et al., 2012; Lo et al., 2017), neuroticism (Okbay, 2016a; Lo et al., 2017; Luciano et al., 2018), well-being (Okbay, 2016a), and depressive symptoms (Okbay, 2016a)]. The highest number of genome-wide significant loci $(n=1,271)$ has been detected for educational attainment (Lee et al., 2018). With respect to mental disorders, the most loci ( $n=145$; Pardinas et al., 2018) have been identified for schizophrenia, for other disorders only one genome-wide significant hit [e.g., anxiety disorders (Otowa et al., 2016), AN (Duncan et al., 2017), conscientiousness (de Moor et al., 2012), and tiredness (Deary, 2018)] has been described.

To address the association of obesity with mental disorders, both clinical and population-based studies have been conducted cross-sectionally and longitudinally (Richardson et al., 2003; Patalay and Hardman, 2019). Particularly well analyzed are associations between obesity and depression (Luppino et al., 2010; Berkowitz and Fabricatore, 2011; Mühlig et al., 2015; Milaneschi et al., 2019) and ADHD (Cortese et al., 2016; Nigg et al., 2016), respectively. For obesity and MDD, a bidirectional, partly sex dependent relationship applies, entailing that obesity can precede MDD and vice versa (Luppino et al., 2014; Mühlig et al., 2015; Coryell et al., 2016). An association with obesity was also reported for BD and schizophrenia (Gurpegui et al., 2012). Because of the adverse effect of weight gain inherent to several psychotropic drugs, especially atypical antipsychotics (Almandil et al., 2013), a reliable assessment of the respective associations can only be based on drug-naïve or previously untreated patients (Gurpegui et al., 2012). A meta-analysis for BMI and WHR in never or minimally treated patients with psychosis or schizophrenia found lower BMI and elevated WHR in patients compared to healthy controls. No differences in WC were observed (Shah et al., 2019).

Observed clinical relationships between anthropometric traits BMI, obesity, body composition, and fat distribution have partially been supported by the detection of significant genetic correlations; in addition, genetic correlations have gone beyond established clinical associations. Significant negative genetic correlations have been described for BMI (or obesity) with educational attainment, intelligence, and neuroticism as well as with schizophrenia and BD. Positive genetic correlations have been found between BMI (or obesity) and ADHD and depression (Bulik-Sullivan et al., 2015; Zheng, 2017; Hill et al., 2018; Richardson et al., 2019b) ${ }^{1}$ : (a) The negative genetic correlations between body fat percentage (BF\%) and educational attainment and schizophrenia should also be highlighted (Richardson et al., 2019b) ${ }^{1}$; (b) At the functional level, several genes coding for proteins involved in synaptic function have been associated with substance use, obesity-related traits, responses to mental and physical stress, heart rate, and blood pressure measurements (Nikpay et al., 2012).

Thus, complex anthropometric and mental phenotypes may reveal extensive overlapping genetic factors. Research is clearly warranted to identify genetic variation involved both in the (1) regulation of body weight, body composition, and body fat distribution and (2) in mental disorders/traits. Particularly, body fat distribution is of great importance, because visceral fat (abdominal obesity) is the main factor underlying the clinical association between obesity and hypertension and type 2 diabetes mellitus (Snijder et al., 2006). Because stress is presumed to play an important role in the development of abdominal obesity (Björntorp, 2001; Geiker et al., 2018; van der Valk et al., 2018), as well as in the pathogenesis of mental disorders (Chrousos, 2009), a genetic link especially with body fat distribution may be particularly pronounced.

The purpose of the current study was to perform for the first time a systematic look-up analysis to assess the potential overlap of genome-wide significant loci for a range of anthropometric traits with mental disorders/traits. Methodologically similar to our previous study, which assessed the role of metabolite SNPs in mental disorders/traits (Hebebrand et al., 2018), we initially identified all genome-wide significant SNPs for the anthropometric traits. Look-ups were subsequently performed in GWAS data sets for 15 mental disorders/traits. For our initial look-up analysis we used data from the GWAS catalog in December 2017. We aimed for more detailed analyses in followup studies. Thus, to keep up with the rapid pace in the GWAS field, we have used the latest data set for each phenotype for our post hoc analyses [effect sizes and directions, Mendelian randomization; e.g., for BMI (Yengo et al., 2018), body fat distribution (Pulit et al., 2019) or schizophrenia (Pardinas et al., 2018), and MDD (Wray et al., 2018)].

\footnotetext{
${ }^{1}$ http://mrcieu.mrsoftware.org/PRS_atlas/
} 
The term "mental disorders" refers to disorders delineated in DSM-5 (American Psychiatric Association, 2013). Mental traits refer to quantitative personality/character, behavioral and socio-psychological traits. We use the overarching term mental phenotypes to refer to both mental disorders and mental traits. Apart from the genetic contribution, there are strong environmental, behavioral, and social components to some of these phenotypes (Meyer-Lindenberg and Tost, 2012).

To exemplarily investigate the potential causality of SNPs for body fat distribution in schizophrenia (and vice versa) in post hoc analyses, we performed a two-sample Mendelian randomization (MR) analysis with $\mathrm{WHR}_{\mathrm{adjBMI}}$ as exposure and the risk for schizophrenia as outcome. In the reverse $M R$ we used the risk of schizophrenia (Richardson et al., 2019a) as exposure and WHR $_{\text {adjBMI }}$ as outcome.

\section{MATERIALS AND METHODS}

\section{Look-Ups}

\section{Selection of Relevant SNPs}

Genome-wide significant SNPs $\left(P \leq 5 \times 10^{-8}\right)$ associated with (i) BMI (obesity), (ii) body composition, and/or (iii) body fat distribution (Figure 1, search terms in Supplementary Table S1) were extracted from the GWAS Catalog version v1.02 (Welter et al., 2014) on 12 December 2017. GWAS Catalog provides uniform data sets (positions, genes, and systematized information on the studies).

The identified studies were independently reviewed by three authors (LL, TP, and JH) in order to exclude anthropometric traits that did not fit the purpose of the analysis. Single different ratings were resolved by consensus. This selection process resulted in the exclusion of some traits from further analyses with a focus on a disease (other than obesity), e.g., BMI in smokers, body mass in chronic obstructive pulmonary disease, BMI (change over time) in cancer, and osteoporosis.

The look-up of the identified SNPs was performed in March 2018 in publically available GWAS data sets for mental phenotypes, taking into account only studies that had identified at least one genome-wide significant locus as previously suggested (Hebebrand et al., 2018). We added a more recent dataset for MDD because of the great importance of this disorder. Based on different consortia the following studies were included: (a) the Psychiatric Genetics Consortium (PGC) ${ }^{3}$ : schizophrenia (Ripke, 2014), ADHD (Demontis et al., 2018), BD (Sklar, 2011), and ASD (Autism Spectrum Disorder Working Group of the Psychiatry Genomics Consortium, 2015; downloaded between 22 November 2016 and 12 February 2017), AN (Duncan et al., 2017; 26 September 2017), MDD (Wray et al., 2018; 2 May 2018); (b) the Social Science Genetic Association Consortium (SSGAC) ${ }^{4}$ : educational attainment (Okbay, 2016b), neuroticism, well-being, and depressive symptoms (Okbay, 2016a; 22 November to 12 December 2016); (c) Anxiety Neuro Genetics Study: anxiety

${ }^{2}$ http://www.ebi.ac.uk/gwas/

${ }^{3}$ https://www.med.unc.edu/pgc/

${ }^{4}$ https://www.thessgac.org/
Search in GWAS Catalog for BMI, body composition and fat distribution traits:

89 studies, 5263 associations, 49 catalog traits

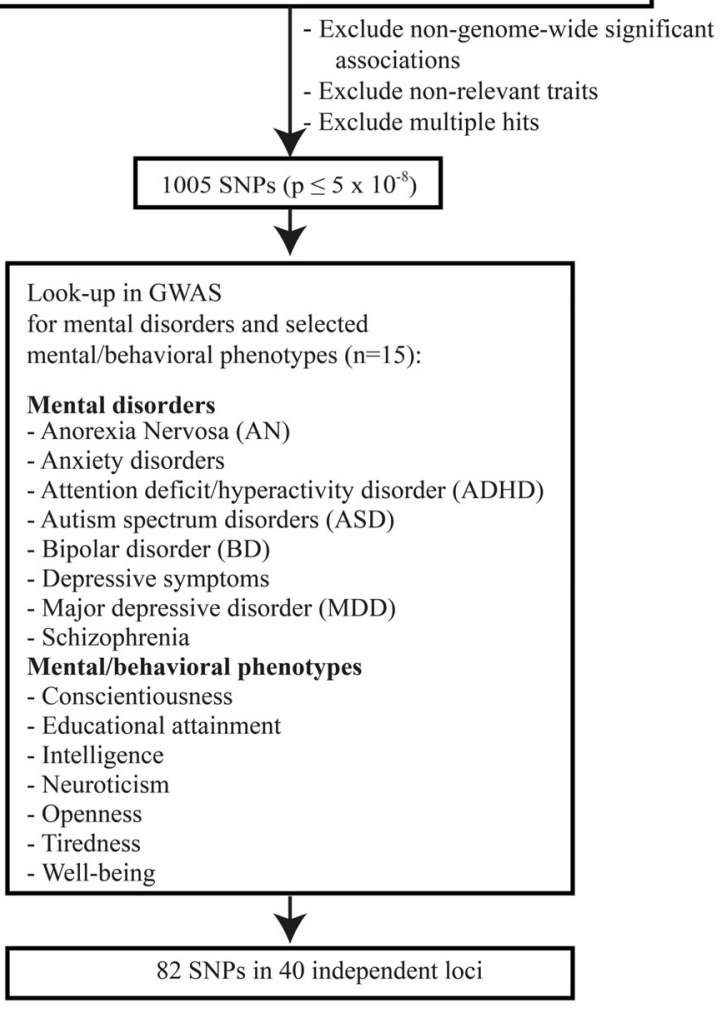

FIGURE 1 | Flow chart of the conducted look-up analysis.

disorder (Otowa et al., 2016; 28 November 2016), (d) Complex Treat Genetic Lab (GTCLab): intelligence (Sniekers et al., 2017; 29 June 2017); (e) The Genetics of Personality Consortium (GPC): conscientiousness and openness (de Moor et al., 2012; 27 September 2017); (f) The Centre for Cognitive Ageing and Cognitive Epidemiology (CCACE): tiredness (Deary, 2018; 19 October 2017; Supplementary Table S3).

To define independent loci we used a distance criterion of $\pm 500 \mathrm{~kb}$ surrounding the middle SNP of a chromosomal locus (Locke et al., 2015). Consecutive numbers were assigned to each locus (Supplementary Table S4; for MDD loci see Supplementary Table S5). Furthermore, linkage disequilibrium $\left(r^{2}\right)$ was checked within each locus and, in case of linkage equilibrium between two SNPs $\left(D^{\prime}<0.01, r^{2}<0.01\right)^{5}$, these were regarded as independent loci.

Independent data sets for confirmatory look-up analyses were available for (a) educational attainment (Davies et al., 2016) [ $n=111,114$, downloaded on 4 December 2017 (Leslie et al., 2014), based on data from the CHARGE and COGENT consortia, and UK Biobank]. These study groups are independent of the 65 study groups included in the study of Okbay (2016b); and

\footnotetext{
${ }^{5}$ https://ldlink.nci.nih.gov/(Machiela and Chanock, 2015)
} 
(b) neuroticism (Luciano et al., 2018; UK Biobank sample size; $n=329,821$; 17 April 2018; Supplementary Table S3).

Genome-wide association studies for mental phenotypes and anthropometric traits have been conducted with study groups that mainly included people of European descent. However, single anthropometric studies were performed in Japanese (Okada et al., 2012; Akiyama et al., 2017), African (Monda et al., 2013; Graff et al., 2017), and East-Asian populations (Wen et al., 2014).

To provide the reader with a synopsis, Tables $\mathbf{1}, \mathbf{2}$ were derived from Supplementary Tables S4, S5, which illustrate the complete results of the look-ups. Tables 1, 2 depict one representative SNP for each study-wide significant locus, which in case of loci tagged by $>1$ SNP was identified by (1) if applicable, the highest number of mental phenotypes hits $\left(P<4.98 \times 10^{-5}\right)$, (2) among these SNPs the one with the lowest $P$-value was chosen.

Based on supplementary files and summary statistics effect directions of the alleles for mental phenotypes and the anthropometric traits were described for each representative SNP $\left(P<4.98 \times 10^{-5}\right)^{6,7}$. In addition, the effect directions of the alleles were included for each anthropometric trait, for which that particular locus showed at least one hit. For this purpose, summary statistics for $\mathrm{HC}, \mathrm{WC}$, WHR, $\mathrm{HC}_{\text {adjBMI}}$,

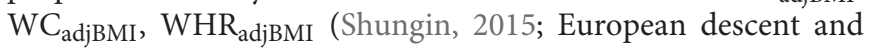
sex-combined), BF\% (Lu et al., 2016), and BMI (European descent; Locke et al., 2015; Yengo et al., 2018) were used.

If a SNP for BMI was found only in a GWAS study based on participants of non-European descent, we reviewed the effect directions and allele frequencies in GWAS of European descent (Locke et al., 2015; Yengo et al., 2018). Summary statistics were downloaded on 30 January 2018 for BMI (Locke et al., 2015) on 14 February 2019, for BMI (Yengo et al., 2018) on 26 February 2019 for WC, WHR, $\mathrm{HC}_{\text {adjBMI}}, \mathrm{WC}_{\text {adjBMI}}$, and WHR adjBMI (Shungin, 2015); for BF\% (Lu et al., 2016) on 26 February $2019^{8,9}$.

\section{Statistical Analyses}

To control for the overall type I error rate in the look-up, Bonferroni correction was applied. We assumed a univariate multiple regression model for each mental phenotype and independence of SNPs. This entailed a Bonferroni correction for 1,005 tests (1,005 SNPs), resulting in a threshold $P$-value of $4.98 \times 10^{-5}$. We did not prune SNPs in high LD to adhere to a conservative approach. For confirmatory analyses, the same $P$-value $\left(4.98 \times 10^{-5}\right)$ was considered as significant.

\section{Mendelian Randomization}

We performed a Mendelian randomization study to exemplarily investigate the causality between body fat distribution and schizophrenia. Body fat distribution data were obtained from the latest GWAS analysis (Pulit et al., 2019; 694,649 individuals), which explained about $3.9 \%$ of the variance in $\mathrm{WHR}_{\text {adjBMI }}$. As

\footnotetext{
${ }^{6}$ https://www.snpedia.com/index.php/SNPedia

${ }^{7}$ https://www.ncbi.nlm.nih.gov/snp

${ }^{8}$ https://portals.broadinstitute.org/collaboration/giant/index.php/GIANT_ consortium_data_files

${ }^{9} \mathrm{ftp} / / / \mathrm{ftp} . e b i . a c . u k /$ pub/databases/gwas/summary_statistics/LuY_26833246_ GCST003435
}

data source for the risk for schizophrenia the GWAS analysis; with 40,675 cases and 64,643 controls (145 independent loci) was used (Pardinas et al., 2018). SNP-based heritability was estimated at 24\% (Pardinas et al., 2018; Pettersson et al., 2019). We conducted a two-sample MR analysis with WHR as risk factor (exposure) and schizophrenia as outcome variable and the reverse analysis. In order to conduct a MR study, three core assumptions must be fulfilled (Haycock et al., 2016; König and Greco, 2018). Therefore, we conducted an F-test to test the weakness of the instrument (Del Greco et al., 2015; Burgess et al., 2017). We examined horizontal pleiotropy by estimating the intercept of Egger's regression (Burgess and Thompson, 2017). To test heterogeneity of the instrument variable, we used Cochran's Q-statistic.

We carried out MR using two statistical methods to estimate overall ratio effects: the inverse-variance weighted method (IVW) and the MR-Egger method. IVW assumes that the all ratio estimates provide independent evidence on the causal effect and there is no pleiotropic effect. So IVW assumes that all genetic variants are valid instrumental variables. There is no intercept term in the regression model (Burgess and Thompson, 2017). In MR-Egger the intercept term is estimated as part of the analyses. The intercept term can be interpreted as the average pleiotropic effect of a genetic variant included in the analyses. A non-zero intercept from MR-Egger shows that there is directional pleiotropy, or that instrumental variable assumptions are violated, or both (Burgess and Thompson, 2017). Consideration of MR-Egger estimates and intercept terms, and comparison of IVW and MR-Egger estimates is helpful to interpret results of a MR analysis (Burgess and Thompson, 2017).

We performed a power analysis to estimate whether our analysis, given sample size, proportion of cases in the study and the proportion of variance explained, is sufficient to detect a true casual effect (Brion et al., 2013). In order to investigate the relationship between study accuracy and effect size, we created a funnel plot (Haycock et al., 2016; Katikireddi et al., 2018). To examine whether an individual data point (SNP) has a large influence on the regression coefficients, we calculated the IVW regression by leaving each genetic variant out in turn (Burgess and Thompson, 2017). To visualize the results, forest and scatter plots were used. We also performed MR with Robust Adjusted Profile Score (RAPS), which estimator is more robust to pleiotropy (Zhao et al., 2019). All tests were performed using the software "R," version 3.5.2 and R-packages for performing 2-sample $\mathrm{MR}^{10}$ and RAPS. ${ }^{11,12}$

\section{RESULTS}

\section{Look-Ups}

A total of 1,005 genome-wide significant $\left(P \leq 5 \times 10^{-8}\right)$ SNPs from 56 studies were identified for BMI/obesity, body

\footnotetext{
${ }^{10} \mathrm{https} / /$ github.com/MRCIEU/TwoSampleMR

${ }^{11}$ https://cran.r-project.org/web/packages/mr.raps/index.html

${ }^{12} \mathrm{https} / /$ github.com/
} 


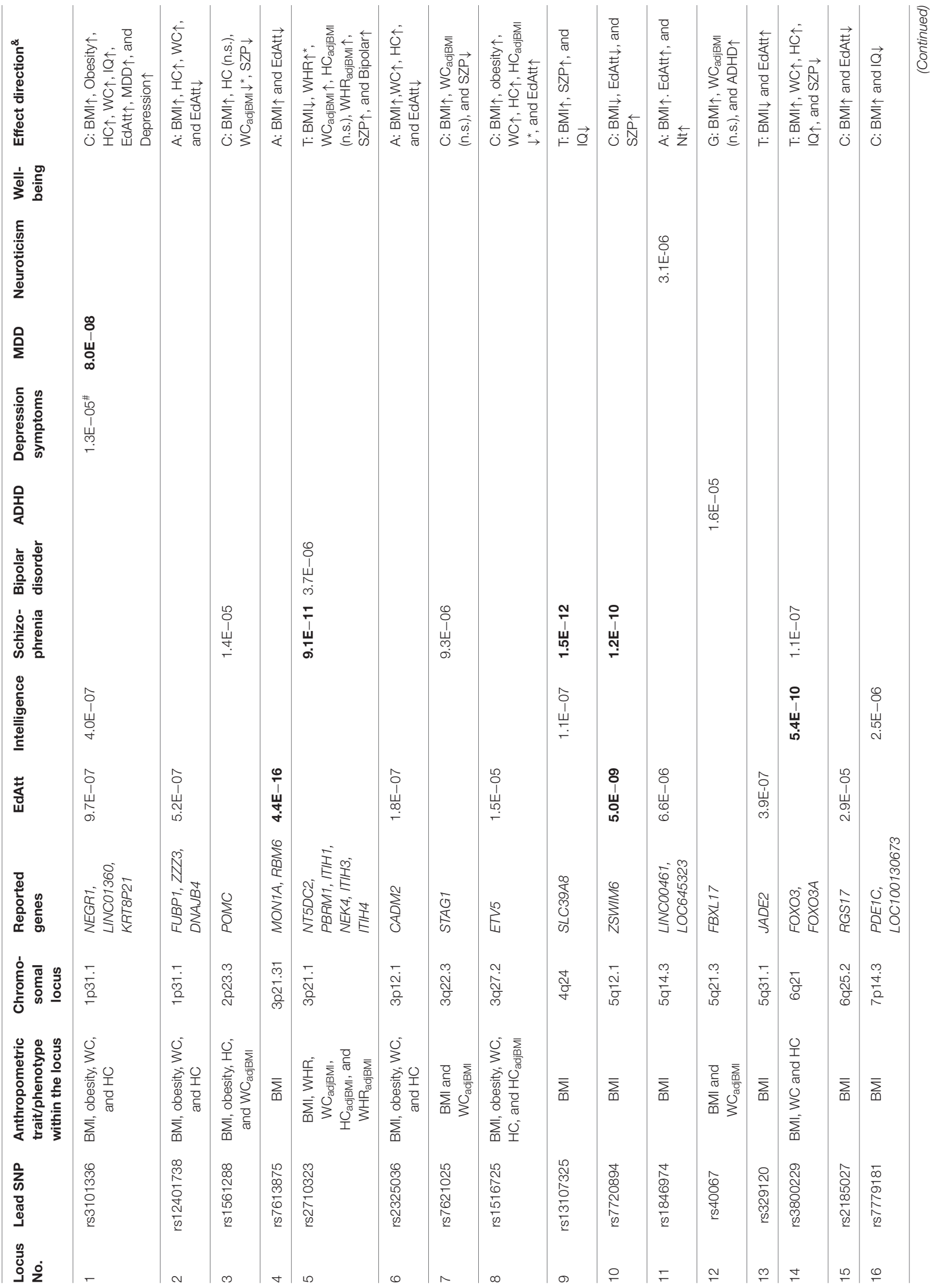




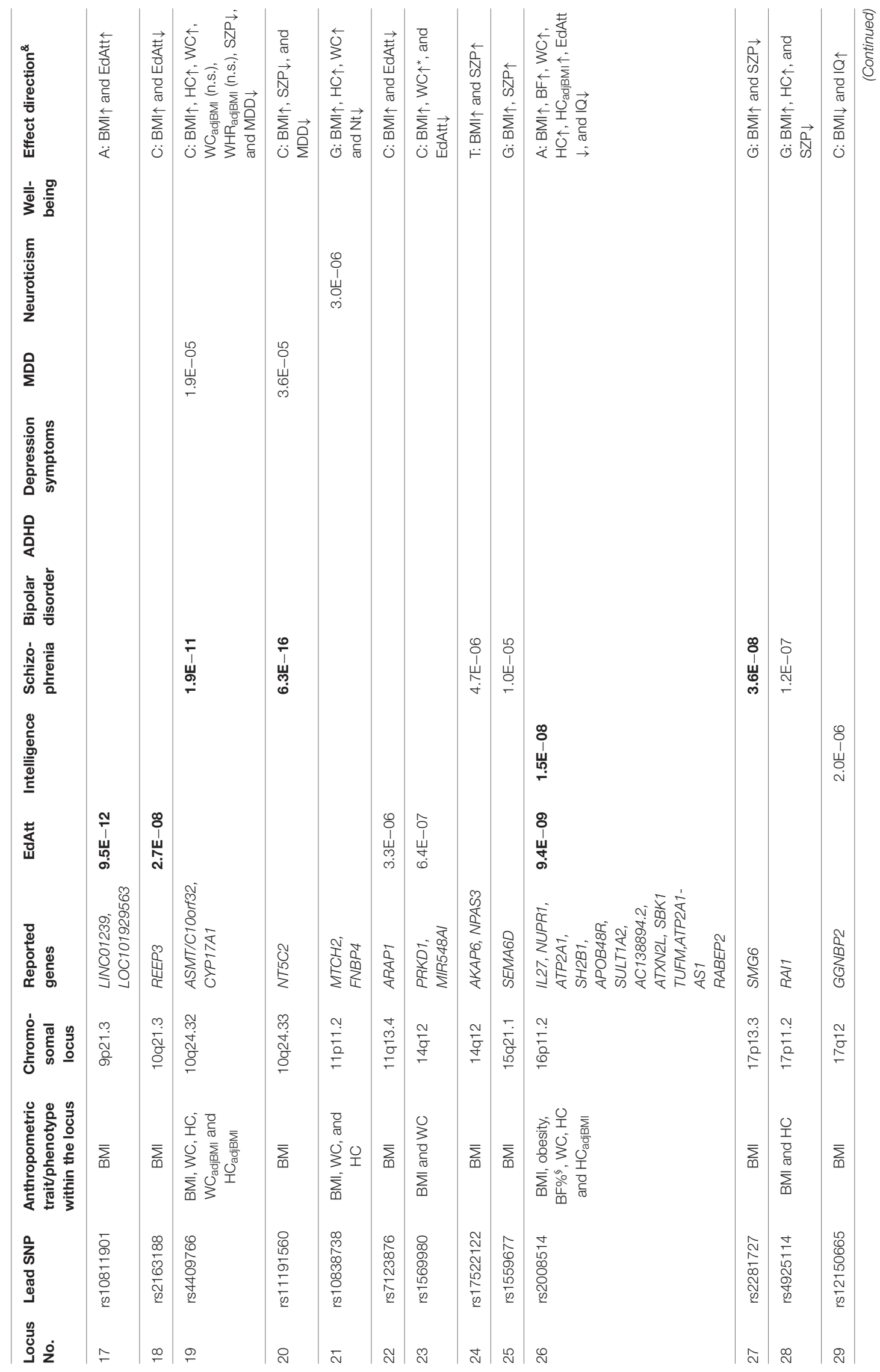




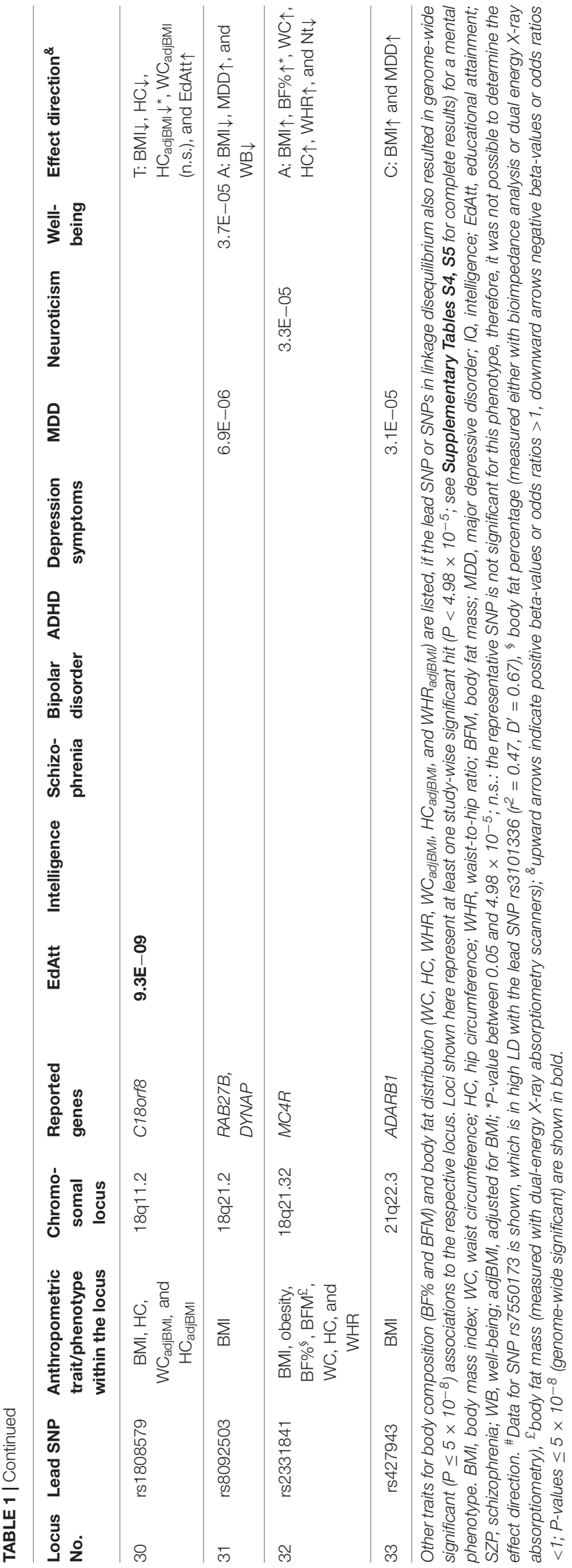

composition, and/or body fat distribution (Supplementary Table S2). Upon Bonferroni correction for multiple testing $\left(P<4.98 \times 10^{-5}\right), 82 \mathrm{SNPs}$ at 40 genomic loci were associated with one or more mental phenotypes (Tables 1, 2; see Supplementary Tables S4, S5 for complete overview), among which the four mental phenotypes educational attainment (16 loci), intelligence (7 loci), schizophrenia (18 loci), and MDD (5 loci) figured prominently. Single loci only were detected for BD, neuroticism, ADHD, depressive symptoms, and well-being. No hits were detected for the remaining six phenotypes (ASD, AN, anxiety, tiredness, conscientiousness, and openness).

For eleven loci, significant hits $\left(P<4.98 \times 10^{-5}\right)$ for more than one mental phenotype - mainly encompassing educational attainment, intelligence and schizophrenia - were identified. Locus 1p31.1 (rs3101336, rs7550173, and comprising NEGR1) was significantly associated with the maximal number of four phenotypes (educational attainment, intelligence, depressive symptoms, and MDD).

The seven of the 40 loci that were not identified via BMI SNPs pertained to different measures of body fat distribution adjusted for BMI (WC $\mathrm{Wdj}_{\mathrm{BMI}}, \mathrm{HC}_{\mathrm{adj} B M I}$, and/or WHR adjBMI ; Table 2). All but one locus were associated with schizophrenia.

We analyzed effect directions of all alleles of significant SNPs $\left(P<4.98 \times 10^{-5}\right)$. For the SNPs in Table 1, we looked up the effect directions for all phenotypes in respective locus (BMI, body composition, body fat distribution, and mental phenotypes) in the respective summary statistics (Locke et al., 2015; Shungin, 2015; Lu et al., 2016; Yengo et al., 2018). As expected, the effect directions were always identical for BMI, obesity, non-adjusted $\mathrm{HC}$ and WC, respectively.

Table 3 provides an overview of the effect directions of all detected significant $\left(P<4.98 \times 10^{-5}\right)$ SNP associations separated according to associations with BMI (irrespective of additional associations to other traits; for loci see Table 1) or with unique associations pertaining to BMI adjusted measures (for loci see Table 2). Opposite effect directions of alleles associated with a higher BMI were observed twice as often for educational attainment, intelligence, and schizophrenia than concordant directions.

\section{Confirmatory Analyses}

We performed confirmatory look-up analyses for educational attainment and neuroticism in the most recent and independent GWAS datasets for SNPs that fulfilled our $P$-value threshold of $P<4.98 \times 10^{-5}$. No independent data sets were available for other mental phenotypes (Supplementary Table S3). Twenty two of the 38 SNPs for educational attainment revealed $P$-values below $P<4.98 \times 10^{-5}$ (10 of 15 loci). For neuroticism, five of seven SNPs (three of four independent loci) were confirmed in an independent dataset (Supplementary Table S6).

\section{Mendelian Randomization}

We performed a MR analysis to investigate the causal effect of body fat distribution, measured as $\mathrm{WHR}_{\mathrm{adj} B \mathrm{BI}}$, on risk of schizophrenia (for data on the 328 genome wide significant SNPs associated with $\mathrm{WHR}_{\text {adjBMI }}$, see Supplementary Table S9). With $F=85.907$ the $F$-statistic indicates strong instrumental 


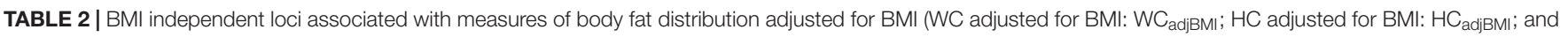
WHR adjusted for BMI: $W_{\text {HCdjBMl }}$; for complete overview of the results see Supplementary Table S4).

\begin{tabular}{|c|c|c|c|c|c|c|c|c|}
\hline Locus No. & Lead SNP & $\begin{array}{l}\text { Anthropometric } \\
\text { trait/phenotype } \\
\text { within the locus }\end{array}$ & Chromosomal locus & Reported genes & EdAtt & Intelligence & Schizophrenia & Effect direction ${ }^{\&}$ \\
\hline 34 & rs615672 & $\begin{array}{l}\text { WC }_{\text {adjBMl }} \text { and } \\
\text { WHR }\end{array}$ & $6 p 21.32$ & HLA-DRB1 & & & $5.7 \mathrm{E}-07$ & $\begin{array}{c}\text { G: } W_{\text {adjBMI } \uparrow}, \\
W H R_{\text {adjBMI} \uparrow} \text {, and } \\
\text { SZP } \uparrow\end{array}$ \\
\hline 35 & rs11156429 & $\begin{array}{c}W_{\text {adjBMl }} \text { and } \\
\mathrm{HC}_{\text {adjBMl }}\end{array}$ & $6 q 16.3$ & HACE1 & & & $2.3 E-06$ & $\begin{array}{c}\mathrm{T}: \mathrm{WC}_{\mathrm{adj} \mathrm{BM}} \uparrow, \\
\mathrm{HC}_{\mathrm{adjBMI} \uparrow} \uparrow \text {, and } \\
\mathrm{SZP} \uparrow\end{array}$ \\
\hline 36 & rs1538170 & $\mathrm{HC}_{\text {adjBMl }}$ & $6 q 22.32$ & CENPW & & 4.9E-05 & & $\mathrm{T}: \mathrm{HC}_{\mathrm{adj} \mathrm{BM}} \uparrow, \mathrm{IQ} \uparrow$ \\
\hline 37 & rs1144 & $W_{\text {adjBMl }}$ & $7 q 22.3$ & SRPK2 & & & $1.3 \mathrm{E}-06$ & $\begin{array}{c}\mathrm{T}: \mathrm{WC}_{\mathrm{adjBM}} \downarrow \text { and } \\
\mathrm{SZP} \downarrow\end{array}$ \\
\hline 38 & rs1727294 & $\mathrm{HC}_{\mathrm{adj} \mathrm{BMI}}$ & $12 q 24.31$ & PITPNM2 & $9.1 E-14$ & $3.4 \mathrm{E}-05$ & $4.7 E-09$ & $\begin{array}{c}\mathrm{A}: \mathrm{HC}_{\text {adjBMI} \uparrow} \uparrow, \\
\mathrm{EdAtt} \uparrow, \mathrm{IQ} \uparrow, \text { and } \\
\mathrm{SZP} \uparrow\end{array}$ \\
\hline 39 & rs4765219 & $\begin{array}{l}\text { WC }_{\text {adjBMl }} \text { and } \\
\text { WHR } \\
\text { adjBMl }\end{array}$ & $12 q 24.31$ & DNAH10, CCDC92 & & & $1.8 \mathrm{E}-05$ & $\begin{array}{c}\mathrm{C}: \mathrm{WC}_{\text {adjBMI }} \uparrow, \\
W H R_{\text {adjBMI }} \uparrow \text {, and } \\
\text { SZP } \downarrow\end{array}$ \\
\hline 40 & rs181553 & $\mathrm{HC}_{\text {adjBMl }}$ & $18 q 21.1$ & $D Y M$ & & & $3.6 E-06$ & $\begin{array}{c}\mathrm{A}: \mathrm{HC}_{\mathrm{adjBMI}} \uparrow \text { and } \\
\mathrm{SZP} \uparrow\end{array}$ \\
\hline
\end{tabular}

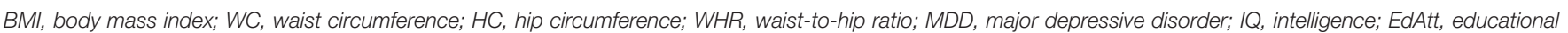

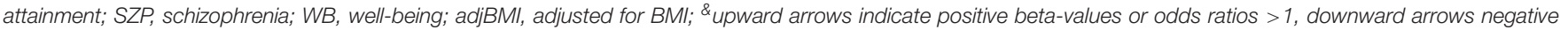
beta-values or odds ratios $<1$; P-values $>5 \times 10-{ }^{8}$ (genome-wide significant) are shown in bold.

variables. After removing palindromic and ambiguous SNPs $(n=9)$, we included 319 SNPs in the analysis. The overall estimates, calculated by IVW or MR Egger, did not reveal associations between $\mathrm{WHR}_{\mathrm{adjBMI}}$ and risk of schizophrenia (IVW, $b=0.0062, P=0.9107$; Supplementary Table S10 and Supplementary Figures S4-S6), although in the single SNP analyses, 47 SNPs showed a significant result (Supplementary Table S11). Sensitivity analyses using the leave-one-out approach confirmed the lack of associations (Supplementary Figure S7). There was no evidence for pleiotropy (MR-Egger intercept $=-0.0003194 ; P=0.8991)$. The heterogeneity test was significant $\left[Q(d f=317)=860.0176, P=1.12 \times 10^{-51}\right]$. Power analysis revealed that our MR analyses had an $80 \%$ power to detect an odds ratio (OR) of 1.09 and $100 \%$ power to detect an OR of 1.28 for schizophrenia (Supplementary Figure S8). The more robust method RAPS also showed no causal effect of body fat distribution on schizophrenia [RAPS main function: beta(hat) $=0.0059, \mathrm{SE}=0.03291$, $P=0.858$; RAPS overdispersed function: beta(hat $)=-0.0744$, $\mathrm{SE}=0.1115, P=0.504]$.

To test reverse causality, we performed an MR analysis where we considered the risk of schizophrenia as a risk factor (for information on the association of 142 SNPs, see Supplementary Table S12) and $\mathrm{WHR}_{\text {adjBMI }}$ as an outcome. After removing palindromic and ambiguous SNPs $(n=4)$, we included 138 SNPs in the analysis. F-test revealed that the instruments were strong $(F=233.896)$. The heterogeneity test was significant $[Q(d f=136)=771.8842$, $\left.P=1.60 \times 10^{-89}\right]$, as was the test for directional horizontal pleiotropy (MR-Egger intercept $=0.00539, \quad P=0.0077)$.
Consequently, we must assume pleiotropy, so that further results must be assumed to be biased. Single SNP analyses revealed that 46 SNPs showed a significant causal effect on WHR $\mathrm{WdjBMI}_{\mathrm{B}}$ with opposite effect directions The overall MR analysis with the Egger-method revealed a causal effect of schizophrenia on WHR adjBMI $(b=-0.073$, $P=0.0098)$, the IVW-method, however, showed no significant effect $(P=0.9296$; Supplementary Tables S13, S14 and Supplementary Figures S9-S11). RAPS method showed no effect [main function: beta(hat) $=0.0007$, $\mathrm{SE}=0.0025$, $P=0.790 ;$ overdispersed function: $\operatorname{beta}($ hat $)=-0.0055$, $\mathrm{SE}=0.0076, P=0.473]$.

\section{DISCUSSION}

We identified 82 SNPs at 40 independent loci for (i) BMI (including obesity), (ii) body composition (BFM and $\mathrm{BF} \%$ ), and/or (iii) body fat distribution (WC, HC, and WHR; adjusted and unadjusted for BMI) concomitantly associated with mental phenotypes. The majority of locus associations were detected with educational attainment $(n=16)$, intelligence $(n=7)$, schizophrenia $(n=18)$, and MDD $(n=5)$. The fact that we found only five loci (1p31.1, 10q24.32, 10q24.33, 18q21.2, and 21q22.3) common for MDD could be due to the relatively low heritability, phenotypic heterogeneity, and multifactorial etiopathogenesis of this disorder (Levinson et al., 2014; Mullins and Lewis, 2017; Milaneschi et al., 2019).

The potential relevance of the identified loci seems to be substantiated by the fact that (a) 15 SNPs loci (seven 
TABLE 3 | Overview of effect directions at the genetic loci for BMl/obesity (see Table 1).

\begin{tabular}{|c|c|c|}
\hline \multirow[t]{2}{*}{ Phenotypes } & \multicolumn{2}{|c|}{$\begin{array}{c}\text { Number of loci with } \\
\text { effect directions in the } \\
\text { same and opposite directions }\end{array}$} \\
\hline & $\uparrow \uparrow$ & $\uparrow \downarrow$ \\
\hline \multicolumn{3}{|l|}{ Educational attainment } \\
\hline $\mathrm{BMI}$ & 5 & 10 \\
\hline Measures adjusted for BMI & 1 & 0 \\
\hline \multicolumn{3}{|l|}{ Intelligence } \\
\hline $\mathrm{BMl}$ & 2 & 4 \\
\hline Measures adjusted for BMI & 2 & 0 \\
\hline \multicolumn{3}{|l|}{ Schizophrenia } \\
\hline $\mathrm{BMI}$ & 4 & 8 \\
\hline Measures adjusted for BMI & 5 & 1 \\
\hline \multicolumn{3}{|l|}{ Bipolar disorder } \\
\hline $\mathrm{BMI}$ & 1 & 1 \\
\hline Measures adjusted for BMI & 0 & 0 \\
\hline \multicolumn{3}{|l|}{ ADHD } \\
\hline $\mathrm{BMl}$ & 1 & 0 \\
\hline Measures adjusted for BMI & 0 & 0 \\
\hline \multicolumn{3}{|l|}{ Depressive symptoms } \\
\hline $\mathrm{BMI}$ & 1 & 0 \\
\hline Measures adjusted for BMI & 0 & 0 \\
\hline \multicolumn{3}{|l|}{ MDD } \\
\hline BMI & 2 & 3 \\
\hline Measures adjusted for BMI & 0 & 0 \\
\hline \multicolumn{3}{|l|}{ Neuroticism } \\
\hline $\mathrm{BMI}$ & 1 & 2 \\
\hline Measures adjusted for BMI & 0 & 0 \\
\hline
\end{tabular}

Anthropometric measures adjusted for BMI WHR adjBMl, WC adjBMl, and $H C_{a d j B M l}$; see Table 2) and mental phenotypes; loci listed for measures adjusted for BMI refer to the total of seven loci uniquely identified via these adjusted measures. Upward arrows indicate positive beta-values or odds ratios $>1$, downward arrows negative beta-values or odds ratios $<1$. BMI, body mass index; all BMI dependent body measures.

for schizophrenia, six for educational attainment, and two for intelligence) at 12 loci met the genome-wide significance threshold and (b) 11 of 18 loci were confirmed for intelligence and neuroticism in independent data sets. Nevertheless, future GWAS and meta-analyses will need to show that our significant hits with $P$-values between $P<4.98 \times 10^{-5}$ and $5 \times 10^{-8}$ indeed represent true findings for the respective mental phenotypes.

Thirty-three loci associated with mental phenotypes were identified via BMI/obesity SNPs (Table 1); for 16 of these loci, the same or additional SNPs identified via other anthropometric traits were co-localized. Thus, as expected based on high correlations between the anthropometric traits (Schneider, 2010) the SNPs for BMI unadjusted traits of body composition and body fat distribution (WC, HC, WHR, BF\%, and BFM) associated with mental phenotypes were always co-associated with the loci identified via BMI SNPs. Only seven loci were unique for BMI adjusted measures of body fat distribution (Table 2). Because of the co-occurrence of nine loci identified both via BMI and measures of body fat distribution adjusted for BMI, we looked up the lead SNPs of these seven loci (Table 2) in the most recent GWAS meta-analyses for BMI (Yengo et al., 2018; Supplementary Table S7); interestingly, all but one locus (locus no. 38: rs1727294) revealed a $P$-value $<0.05$, one of which was even genome-wide significant (locus no. 39: rs12317176). Clearly, the overall frequent co-identification of loci for BMI and BMI adjusted measures of body fat distribution indicates an overlap between BMI and BMI adjusted body fat distribution loci with respect to the respective mental phenotypes.

Recently, Kaur et al. (2018) conducted look-up analyses in several somatic and mental disorders using genome wide significant $\mathrm{WHR}_{\text {adjBMI }}$ SNPs as measurement for body fat distribution/abdominal obesity. In accordance with our results, they found that two WHR adjBMI variants in 3 p21.1 (see locus 5 in Table 1) are associated with schizophrenia (PBMI1 and ITIH1) and BD (ITIH1) (Kaur et al., 2018); our results indicate that $\mathrm{BMI}, \mathrm{HC}_{\mathrm{adj} \mathrm{BMI}}$, and $\mathrm{WC}_{\mathrm{adjBMI}}$, too, are associated with these mental disorders. Additionally, they reported that LOC105375015 (rs2596500) was associated with WHR adjBMI $_{\text {and }}$ schizophrenia, a finding we could not confirm as our analyses did not include proxy SNPs.

There are numerous indications that the clinical association between abdominal obesity and cardiometabolic risk is largely independent of BMI (Després, 2006; van Vliet-Ostaptchouk et al., 2013). For the latest genetic study on body fat distribution (WHR adjBMI; Pulit et al., 2019), the potential for collider bias resulting from conditioning WHR on BMI was analyzed. They found that most $\mathrm{WHR}_{\mathrm{adjBMI}}$ SNPs (311 of 346) are associated with stronger standard deviation effect sizes for WHR than for BMI. Each additional (weighted) $\mathrm{WHR}_{\mathrm{adjBMI}^{-}}$ raising allele was associated with a higher WHR and with a lower BMI (Pulit et al., 2019). We looked up the 346 SNPs associated with $\mathrm{WHR}_{\text {adjBMI }}$ (Pulit et al., 2019) in the recent GWAS for BMI (Yengo et al., 2018): 263 SNPs overlapped between the two data sets (Yengo et al., 2018; Pulit et al., 2019), 20 of which were genome-wide significant for both $\mathrm{WHR}_{\mathrm{adjBMI}}$ and BMI. In light of the high number of SNPs identified in our study that have an effect on both BMI and WHR $_{\text {adjBMI }}$ and the less than $10 \%$ overlap (20/263) of BMI and WHR $_{\text {adjBMI }}$ (Yengo et al., 2018; Pulit et al., 2019). SNPs we postulate that loci having independent effects on both anthropometric traits have a higher probability of playing a role in mental phenotypes.

Our data indicate that the associations between anthropometric traits and mental disorders/traits are heterogeneous as some effects were directionally consistent and for others the risk alleles pointed to opposite directions (Table 3). As expected, effect directions for BMI, obesity, HC, and $\mathrm{WC}$ were consistent for all the respective 16 loci; for the two loci (nos. 5 and 32; Table 1) co-identified by WHR one effect directions was concordant, the other discordant to BMI. The overall associations between genetic risks for different diseases or genetic proportion of variance across all SNPs are expressed with genetic correlations or correlations between genetic risk scores. Small to medium, but nevertheless significant genetic correlations have been detected between BMI and schizophrenia $\left(r_{g}=-0.10, P=1.7 \times 10^{-4}\right)$ (Bulik-Sullivan et al., 2015), BMI and ADHD $\left(r_{g}=0.26, P=3.0 \times 10^{-3}\right)$, $\mathrm{BMI}$ and childhood intelligence $\left(r_{g}=-0.17, P=2.3 \times 0^{-3}\right)$ 
(Bulik-Sullivan et al., 2015), BMI and intelligence $\left(r_{g}=-0.16\right.$, $\left.P=1.4 \times 10^{-16}\right)$ (Hill et al., 2018), BMI and educational attainment $\left(r_{g}=-0.28, P=6.6 \times 10^{-16}\right)$ (Bulik-Sullivan et al., 2015), and BMI and $\mathrm{AN}\left(r_{g}=-0.18, P=3.17 \times 10^{-7}\right)$ (Bulik-Sullivan et al., 2015; Hill et al., 2018). Between WC or WHR and educational attainment genetic correlations of $-0.29\left(P=2.31 \times 10^{-14}\right)$ and $-0.33\left(P=1.34 \times 10^{-18}\right)$ were reported (Bulik-Sullivan et al., 2015). The correlations between genetic risk scores for both schizophrenia and educational attainment with $\mathrm{BF} \%$ were negative $(P<0.05$; Richardson et al., 2019a). We were unable to find studies providing genetic correlations between BMI, body composition, and body fat distribution traits.

For schizophrenia, we identified the strongest overlap with body fat distribution traits adjusted for BMI in our look-up analysis (Table 2). Most clinical studies (Sugawara et al., 2012; Konarzewska et al., 2014; Strassnig et al., 2017), but not all (Kim et al., 2017) have found a higher BF\%, BFM, visceral adipose tissue mass in normal weight patients with schizophrenia as compared to healthy controls. These clinical observational studies either considered antipsychotic medication as a covariable (Strassnig et al., 2017), discuss it as a justification for observed differences (Kim et al., 2017), or mention the inclusion of patients on antipsychotics as a limitation (Sugawara et al., 2012). Besides general limitations of observational studies such as residual confounding, directions of potential causal links between body fat distribution and schizophrenia remain unclear. MR assumes that genetic variants are not confounded by behavioral, socioeconomic, and physiological factors and genetic variants will not be influenced by the onset of disease (Davey Smith and Ebrahim, 2005). Therefore, MR can be regarded as a randomized controlled trial provided by nature. For this reason, we applied MR analysis to the most recent data to test whether there is an overall causal association between BMI adjusted body fat distribution and schizophrenia.

The MR analysis did not reveal evidence for an overarching effect of adjusted body fat distribution on the risk for this mental disorder. The power analysis revealed that our MR had a $100 \%$ power to detect an OR of 1.28 for schizophrenia. This OR is similar as those observed for single SNPs calculated in GWAS: e.g., the T-allele of rs3130820 (the SNP with the highest effect in GWAS by Pardinas et al., 2018) increases the risk of getting a diagnosis of schizophrenia by 1.281 times $(\mathrm{SE}=0.018$, $\left.P=2.12 \times 10^{-44}\right)$; the OR of the T-allele at rs12416331 is $1.157\left(\mathrm{SE}=0.017, P=7.09 \times 10^{-18}\right)$. Thus, the power of our MR was sufficient to detect a clinically relevant risk for schizophrenia. In the reverse analyses about the causal effect of schizophrenia on body fat distribution, the non-zero intercept from MR-Egger indicates that there is the directional pleiotropy or the assumptions for instrumental variable are violated, or both. The Egger method showed a significant effect, but the IVW method showed none. The RAPS method, which is more robust to pleiotropy, showed no effect. Our MR analyses suggest that there is no causal effect of schizophrenia on body fat distribution.

Our study now shows that specific genetic effects at individual loci may well play a role in associations between both BMI adjusted body fat distribution and schizophrenia.
In the following, we provide the reader with an insight into potential functional implications of five of the 40 loci which we

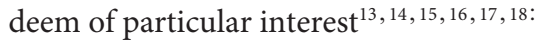

(1) Locus 1p31.1 (rs3101336, rs7550173, NEGR1; locus 1 in Table 1) was significantly associated with both MDD and depressive symptoms, educational attainment, and intelligence. NEGR1 (neuronal growth regulator 1 gene), a well-known obesity-associated gene (Willer, 2009), is associated with white matter integrity (Dennis et al., 2014), and shows high expression in several brain tissues (highest median expression in frontal cortex, Supplementary Figure S1). NEGR1 is a member of the immunoglobulin domain-containing glycosylphosphatidylinositol (GPI)anchored neural cell adhesion molecules (IgLON; Karis et al., 2018) and involved in neural cell communication and synapse formation (Joo et al., 2019). Recently, NEGR1 was also found to play a role in intracellular cholesterol trafficking (Joo et al., 2019). Overall, NEGR1 seems to play a pleiotropic role due to its involvement in synaptogenesis, neuron maturation, and neurite outgrowth, which all represent essential mechanisms for sustaining brain function and development (Pischedda and Piccoli, 2015). Significantly, elevated levels of NEGR1 transcripts were found in the dorsolateral prefrontal cortex of patients with schizophrenia compared to healthy controls (Karis et al., 2018).

(2) CYP17A1 (cytochrome P450 family 17 subfamily a member 1 gene) in 10q24.33 (locus 19 in Table 1) is one of the well-known genes associated with adult hypertension (Levy et al., 2009). We found an association with BMI, WC, HC, $\mathrm{WC}_{\mathrm{adjBMI}}, \mathrm{HC}_{\mathrm{adjBMI}}$, and both schizophrenia and MDD. CYP17A1 has previously been found to be associated with schizophrenia (Ripke, 2014); our detected association is novel for MDD. In the recently published multivariate study on the wellbeing spectrum, CYP17A1 was associated with depressive symptoms and neuroticism (Baselmans et al., 2019). A potentially overarching functional implication of this locus in both somatic and mental disorders is supported (Van Woudenberg et al., 2015) by the fact that in adolescent males but not females, a CYP17A1 SNP was associated with enhanced blood pressure reactivity to mental stress (Van Woudenberg et al., 2015). CYP17A1 is predominantly expressed in adrenal glands (Supplementary Figure S2) and is a bifunctional key regulatory enzyme in the steroidogenic pathway with $17 \alpha$-hydroxylase and 17,20-lyase activity (Yoshimoto and Auchus, 2015). CYP17A1 is essential for the formation of all endogenous androgens (Peng et al., 2019) and many glucocorticoids (Kmetova Sivonova et al., 2017). Key substrates are pregnenolone and

\footnotetext{
${ }^{13}$ www.genecards.org

${ }^{14}$ www.ncbi.nlm.nih.gov/gene

${ }^{15}$ www.uniprot.org (The UniProt Consortium, 2017)

${ }^{16}$ www.omim.org http://ldsc.broadinstitute.org/ldhub/

${ }^{17}$ https://gtexportal.org/home/(Consortium et al., 2017)

${ }^{18}$ https://gene.sfari.org/
} 
progesterone, which are transformed to the androgen precursor's dehydroepiandrosterone and androstenedione (Kim et al., 2016). Several findings indicate a sexrelated susceptibility for schizophrenia with males at higher risk, e.g., earlier age of onset compared to a lower susceptibility for women due to a potentially neuroprotective role of estrogens (Seeman, 1996) via effects on dopamine (DA) neurotransmission (Di Paolo, 1994; Sanchez et al., 2010; Frau et al., 2014; Godar and Bortolato, 2014). It is hypothesized that androgenic metabolites of testosterone, especially dihydrotestosterone (DHT) and androstenedione, may facilitate the development of schizophrenia-related symptoms. Quantitative real-time PCR in post-mortem brain slices of MDD patients showed a significant decrease in mRNA levels of CYP17A1 (Qi et al., 2018) and genetic variants of the rs743572 polymorphism of CYP17A1 were correlated with the intensity of depressive symptoms in post-menopausal women (Loja-Chango et al., 2016), altogether suggesting a role of CYP17A1 in mood disorders.

(3) At the locus 16p.11.2 (see locus 26 in Table 1) higher $\mathrm{BMI}, \mathrm{BF} \%, \mathrm{WC}, \mathrm{HC}, \mathrm{HC}_{\mathrm{adj} \mathrm{BMI}}$, and higher risk for obesity were negatively associated with intelligence and educational attainment in line with previous evidence (Jacquemont et al., 2011). Genetic variations [SNPs, copy number variants (CNVs), and larger deletions/insertions] at 16p.11.2 have been shown to be associated with developmental delay, ASD, ADHD, schizophrenia, $\mathrm{BD}$, epilepsy, intracranial volume, brain development, congenital anomalies, altered satiety response, energy imbalance, underweight, and morbid obesity (McCarthy et al., 2009; Bochukova et al., 2010; Fernandez et al., 2010; Walters et al., 2010; Jacquemont et al., 2011; Volckmar et al., 2012; Zufferey et al., 2012; Egger et al., 2014; Qureshi et al., 2014; Volckmar et al., 2015; Maillard et al., 2016; Giuranna et al., 2018; Martin-Brevet et al., 2018; Sonderby et al., 2018; Niarchou et al., 2019). The consequences of 16p11.2 CNV and larger deletions and duplications on health are broad (Crawford et al., 2019; Niarchou et al., 2019). Because the CNVs are rare (Stefansson et al., 2014), large studies and complex analytical methods are needed to detect them in GWAS (Nothen et al., 2019).

The SNPs we found in $16 \mathrm{p} 11.2$ are assigned to genes IL27, NUPR1, ATXN2L, SH2B1, TUFM, SBK1, SULT1A2, ATP2A1, APOB48R, AC138894.2, ATP2A1-AS1, and RABEP2 (see Supplementary Table S6); however, a total of 41 genes may be affected by the CNV in this region (Stefansson et al., 2014). The protein encoded by IL27 (Interleukin 27) is one of the subunits of a heterodimeric cytokine complex and is in many aspects closely tied to the cytokines IL-12 and IL-23. IL-27 may play a role in the rapid initiation of a response to an inflammatory challenge (Pflanz et al., 2002). Neuro-inflammatory processes as a result of stress-induced immune system activation (Halaris, 2019) are discussed as potential mechanisms in the pathogenesis of mental disorders, e.g., depression (Berk et al., 2013). This hypothesis was recently supported by systems genomics studies (Sharma, 2016) and clinical intervention studies with COX-2 inhibitors and aspirin (Muller, 2019). A negative phenotypical correlation of the level of systemic inflammation biomarkers with general intelligence in children also has been observed (Lee et al., 2016). Inflammation and metabolic risk factors might increase the risk of psychosis in some people (Perry et al., 2019), inflammatory mechanisms may also play a role in the pathophysiology of stress-related and anxiety disorders (Lasselin et al., 2016).

(4) In 3p12.1 we found an association between BMI (obesity) and educational attainment (rs2325036, CADM2; see locus 6 in Table 1). Cell adhesion molecule 2 (CADM2) belongs to the immunoglobulin superfamily and interacts with other cytoskeletal proteins (Biederer, 2006). It is predominantly expressed in the central and peripheral nervous systems (Pellissier et al., 2007; Morris et al., 2019; Supplementary Figure S3) and regulates, e.g., synapse formation (Rathjen et al., 2017). Obesity risk variants (Yan et al., 2018; Morris et al., 2019) were associated with increased CADM1 and CADM2 expression in the hypothalamus with a complex interplay of $C A D M 1$ and CADM2 (Rathjen et al., 2017). Reduced expression promoted a negative energy balance and weight loss in mice (Rathjen et al., 2017). The expression of both $C A D M 1$ and $C A D M 2$ in several neuronal circuits suggests an intertwining of body weight maintenance with motor control, but also behavioral aspects, cognition, and memory (Rathjen et al., 2017). In addition to associations with obesity risk genes (SNP rs13078807 located in the second intron of CADM2) novel associations of CADM2 genetic variants with systolic blood pressure (SBP), CRP levels (Morris et al., 2019), as well as with diverse psychological traits (neuroticism, mood instability, and risk-taking) have been detected. Interestingly, other loci identified by SNPs for BMI and/or body fat distribution in the current study are involved in the immune system, too (Supplementary Table S8): ITIH4 (Murakami et al., 2015; locus 5, 3p21.1; BP, schizophrenia), SLC39A8 (Liu et al., 2013b; locus 9, 4q24; schizophrenia, intelligence), and HLA-DRB1 (Arango et al., 2017; locus 34, 6p21.32; schizophrenia).

(5) We for the first time report an association of $M C 4 R$ (locus 32; Table 1 and Supplementary Table S4) with neuroticism. MC4R is well known for its role in BMI/obesity (Hinney et al., 2013). Evidence from animal models links the MC4R to eating behavior in stress-induced anxiety-like or depression-like behavior, anhedonia, and corticosterone secretion (Lim et al., 2012; Liu et al., 2013a; Sabban et al., 2015). MC4R appears to interact with the SAPAP3 gene regulating compulsive behavior and body weight (Xu et al., 2013). A higher rate of anxiety and compulsiveness in patients with AN might be mediated by the MC4R (Xu et al., 2013). The MC4R (rs17782313-C) has also been associated with elevated scores for emotional eating and craving for food (Yilmaz et al., 2015). Gain of function variants in $M C 4 R$, which have a weight lowering 
effect (Wang et al., 2010), have been associated with binge eating disorder (BED) in overweight subjects. Apart from polymorphisms exerting weak effects on BMI 166 MC4R mutations mostly entailing a loss in function have been identified up to date mainly in obese individuals (Hinney et al., 2013). Apart from obesity, mutation carriers showed increased lean mass, increased linear growth, hyperphagia, hyperinsulinemia, and ADHD (Loos et al., 2008; Albayrak et al., 2011; Hinney et al., 2013).

\section{LIMITATIONS}

Limitations of our study include: (i) we conducted a lookup analysis using summary statistics, so that we could not analyze covariates. (ii) The number of hits was higher for mental phenotypes with higher heritability, higher number of genome-wide significant loci, and/or higher numbers of subjects in the respective GWAS. Therefore, we suspect a type 2 error for phenotypes with a currently low number of identified loci. (iii) We had performed a Bonferroni correction for the 1,005 SNPs. As a drawback true associations may have been missed. On the other hand, we did not correct for the number of analyzed traits. (iv) We were unable to systematically consider the ancestry/ethnicity of the study groups comprising the diverse GWAS. However, as most data were derived from individuals of European descent this should represent a minor issue only. For those loci, which were detected in non-European study groups, we checked $P$-values and effect directions in recent GWAS conducted in individuals of European descent. (v) The progress in GWAS research leads to the release of many new analyses every year. For most phenotypes the selected GWAS data sets are the most recent ones; it was, however, not possible to include the most recent analyses, e.g., for

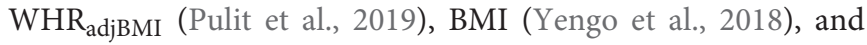
schizophrenia (Pardinas et al., 2018) in our look-up analyses. We were, however, able to use these most recent studies for MR-analysis and additional look-ups. (vi) Sex- and age-stratified analyses were not possible as the respective data sets were not available for all traits despite evidence for differences in genetic regulation for BMI and body fat distribution depending on age and sex (van Vliet-Ostaptchouk et al., 2013; Winkler et al., 2015; Pulit et al., 2019; Rask-Andersen et al., 2019). For body shape (WHR $\mathrm{WdjBMI}_{\mathrm{B}}$ ), age effects were not found (Winkler et al., 2015). (viii) It needs to be noted that across the literature for body fat distribution the term "adjusted for BMI" is not defined uniformly. It can either mean that the measurement (WC, HC, and WHR) is directly adjusted for BMI or that the estimation of the SNP effects in a GWAS was adjusted for BMI. For future GWAS a clear definition of the term "adjusted for BMI" is needed. (viii) We defined loci mainly via a central $\mathrm{SNP}$ and a surrounding region $( \pm 500 \mathrm{~kb})$. However, some loci might actually represent more than one independent locus. We attempted to detect independent hits by analysis of linkage disequilibrium; nevertheless, independent SNPs within a given locus may have escaped detection. (ix) A clinically relevant aspect of the relationship between BMI and mental disorders is the susceptibility to weight gain as a side effect of treatment with especially atypical antipsychotic drugs. Because the GWAS data for the anthropometric traits are mostly based on "healthy" individuals, large scale confounding via use of psychotropic drugs a priori appears unlikely. (x) Study samples for some of the examined phenotypes are not completely independent. Sample overlap can potentially affect the results of cross-trait analysis. Since our analyses were conducted using summary statistics it is not possible to determine an exact number of overlapping participants between the underlying GWAS used in our analyses. To assess whether the overlap can be assumed, we looked at whether GWAS studies included in our analysis used data from the same study groups.

The GWAS study on body fat distribution (Pulit et al., 2019), which we used for our MR analysis included a total of 29 study groups of the PGC and of UK Biobank. Four cohorts (B58C-WTCCC, TWINSUK, EGCUT, and KORA) are also mentioned as sources of controls for GWAS for schizophrenia (Pardinas et al., 2018), which we used as outcome phenotype. For cases with schizophrenia CLOZUK1, CLOZUK2, and CardiffCOGS cohorts and the PGC cohorts were used. These study groups were apparently not used for the GWAS on body fat distribution (Pulit et al., 2019).

Two-sample MR assumes independent samples. Violation of this assumption will result in increased Type 1 error and biased effect estimates (Burgess et al., 2016). For a continuous outcome, bias due to sample overlap is a linear function of the proportion of overlap between the samples with IVW method. Bias due to sample overlap may be more serious for the MREgger method. In a simulation analyses for binary outcome with sample overlap in the controls only - as it is the case in our study - there was no detectable bias in the IV estimates even with extremely weak instruments, nor was there any inflation of type 1 error rates (Burgess et al., 2016). Accordingly, in our MR study, the sample overlap between controls of schizophrenia GWAS and study participants for GWAS on risk variable (i.e., WHR adjusted for BMI) do not lead to a bias for estimated causality in two sample MRs with binary outcome (Burgess et al., 2016).

Sample overlap can also influence the results of the look-up analysis. As an example we compared studies that were included in the GWAS on BMI (158 studies in total; Locke et al., 2015) and studies that were included in the GWAS on educational attainment (63 studies in total; Okbay, 2016b). This comparison revealed that nineteen studies were the same.

We also checked the overlapping of the study groups included in the GWAS on BMI (GIANT; Locke et al., 2015) and in the GWAS on schizophrenia (PGC; Ripke, 2014). It is possible that a certain number of controls for the GWAS on schizophrenia were the same individuals included in GWAS on BMI (overlapping cohorts were EGCUT, MIGen, WTCCC, KORA, HRN, MGS, and deCODE).

The sample overlap between the GWAS on psychiatric disorders due to comorbidity should also be considered. This topic had previously been investigated using data from PGC (LeBlanc et al., 2018). They found that the overlap was less than $2 \%$ for eight psychiatric disorders. Data sets in the study were 
the same as in our study for four disorders (ADHD, AN, MDD, and schizophrenia). Nevertheless, we can probably assume that the sample overlap is of a similar magnitude for studies included in our analyses.

\section{CONCLUSION}

We identified several loci that were associated with BMI, body composition and/or body fat distribution, and mental disorders/traits. The SNPs for body composition and body fat distribution (WC, $\mathrm{HC}, \mathrm{WHR}, \mathrm{BF} \%$, and $\mathrm{BFM}$ ) associated with mental disorders/traits were all simultaneously associated with BMI. Hence, an isolated effect of body fat distribution on mental phenotypes implied by clinical and epidemiological studies (Luppino et al., 2010; Berkowitz and Fabricatore, 2011; Gurpegui et al., 2012; Luppino et al., 2014; Mühlig et al., 2015; Cortese et al., 2016; Coryell et al., 2016; Nigg et al., 2016), was not substantiated. In the MR analysis, we did not detect a general causal effect of body fat distribution on schizophrenia. Our study has confirmed some known cross-phenotype associations (Brainstorm et al., 2018) but has also given some new clues for genetic loci involved in both anthropometric traits and mental phenotypes.

\section{DATA AVAILABILITY STATEMENT}

The datasets for this article are publicly available (see section "Materials and Methods").

\section{AUTHOR CONTRIBUTIONS}

All authors contributed to the writing of the manuscript and approved of the final version. TP planned and supervised the

\section{REFERENCES}

Akiyama, M., Okada, Y., Kanai, M., Takahashi, A., Momozawa, Y., Ikeda, M., et al. (2017). Genome-wide association study identifies 112 new loci for body mass index in the Japanese population. Nat. Genet. 49, 1458-1467. doi: 10.1038/ng. 3951

Albayrak, Ö., Albrecht, B., Scherag, S., Barth, N., Hinney, A., and Hebebrand, J. (2011). Successful methylphenidate treatment of early onset extreme obesity in a child with a melanocortin-4 receptor gene mutation and attention deficit/hyperactivity disorder. Eur. J. Pharmacol. 660, 165-170. doi: 10.1016/j. ejphar.2010.12.023

Almandil, N. B., Liu, Y., Murray, M. L., Besag, F. M. C., Aitchison, K. J., and Wong, I. C. K. (2013). Weight gain and other metabolic adverse effects associated with atypical antipsychotic treatment of children and adolescents: a systematic review and meta-analysis. Pediatr. Drugs 15, 139-150. doi: 10.1007/s40272-0130016-6

American Psychiatric Association, (2013). Diagnostic and Statistical Manual of Mental Disorders (DSM-5 $\left.{ }^{\circledR}\right)$. Washington, DC: American Psychiatric Pub.

Arango, M. T., Perricone, C., Kivity, S., Cipriano, E., Ceccarelli, F., Valesini, G., et al. (2017). HLA-DRB1 the notorious gene in the mosaic of autoimmunity. Immunol. Res. 65, 82-98. doi: 10.1007/s12026-016-8817-7

Autism Spectrum Disorder Working Group of the Psychiatry Genomics Consortium, (2015). Dataset: PGC-ASD Summary Statistics from a MetaAnalysis of 5,305 ASD-Diagnosed Cases and 5,305 Pseudocontrols of European Descent (based on similarity to CEPH reference genotypes). Available at: http: //www.med.unc.edu/pgc/results-and-downloads (accessed March, 2015). project, performed the analyses, discussed the results and wrote the manuscript; LN performed the analyses and drafted the first version of the manuscript; B-HL advised and reviewed the MR analysis; $\mathrm{AH}$ interpreted the results and was part of the writing team; JA discussed the results and was part of the writing team; LT contributed to analyses; RN performed the MR analyses; CA provided critical input on early versions of the manuscript; LL discussed the results and commented on the manuscript; JH planned and supervised the project, discussed the results, and wrote/finalized the manuscript.

\section{FUNDING}

We thank the following sources for funding: the German Ministry for Education and Research (National Genome Research Net-Plus 01GS0820), the German Research Foundation (DFG; HI865/2-1), and the University Hospital Essen. AH was supported by the "Landesprogramm für Geschlechtergerechte Hochschulen - Programmstrang Förderung von Denominationen in der Genderforschung." The Genotype-Tissue Expression (GTEx) Project was supported by the Common Fund of the Office of the Director of the National Institutes of Health, and by NCI, NHGRI, NHLBI, NIDA, NIMH, and NINDS. The data used for the analyses described in this manuscript were obtained from the GTEx Portal on between $03 / 05 / 18$ and $06 / 26 / 18$.

\section{SUPPLEMENTARY MATERIAL}

The Supplementary Material for this article can be found online at: https://www.frontiersin.org/articles/10.3389/fgene. 2020.00373/full\#supplementary-material

Baselmans, B. M. L., Jansen, R., Ip, H. F., van Dongen, J., Abdellaoui, A., van de Weijer, M. P., et al. (2019). Multivariate genome-wide analyses of the well-being spectrum. Nat. Genet. 51, 445-451. doi: 10.1038/s41588-018-0320-8

Berk, M., Williams, L. J., Jacka, F. N., O’Neil, A., Pasco, J. A., Moylan, S., et al. (2013). So depression is an inflammatory disease, but where does the inflammation come from? BMC Med. 11:200. doi: 10.1186/1741-7015-11-200

Berkowitz, R. I., and Fabricatore, A. N. (2011). Obesity, psychiatric status, and psychiatric medications. Psychiatr. Clin. North Am. 34, 747-764. doi: 10.1016/j. psc.2011.08.007

Biederer, T. (2006). Bioinformatic characterization of the SynCAM family of immunoglobulin-like domain-containing adhesion molecules. Genomics 87, 139-150. doi: 10.1016/j.ygeno.2005.08.017

Björntorp, P. (2001). Do stress reactions cause abdominal obesity and comorbidities? Obes. Rev. 2, 73-86. doi: 10.1046/j.1467-789x.2001.00027.x

Bochukova, E. G., Huang, N., Keogh, J., Henning, E., Purmann, C., Blaszczyk, K., et al. (2010). Large, rare chromosomal deletions associated with severe early-onset obesity. Nature 463, 666-670. doi: 10.1038/nature08689

Brainstorm, C., Anttila, V., Bulik-Sullivan, B., Finucane, H. K., Walters, R. K., Bras, J., et al. (2018). Analysis of shared heritability in common disorders of the brain. Science 360:eaa8757. doi: 10.1126/science.aap8757

Brion, M. J., Shakhbazov, K., and Visscher, P. M. (2013). Calculating statistical power in Mendelian randomization studies. Int. J. Epidemiol. 42, 1497-1501. doi: 10.1093/ije/dyt179

Bulik-Sullivan, B., Finucane, H. K., Anttila, V., Gusev, A., Day, F. R., Loh, P. R., et al. (2015). An atlas of genetic correlations across human diseases and traits. Nat. Genet. 47, 1236-1241. doi: 10.1038/ng.3406 
Burgess, S., Davies, N. M., and Thompson, S. G. (2016). Bias due to participant overlap in two-sample Mendelian randomization. Genet. Epidemiol. 40, $597-$ 608. doi: $10.1002 /$ gepi.21998

Burgess, S., Small, D. S., and Thompson, S. G. (2017). A review of instrumental variable estimators for Mendelian randomization. Stat. Methods Med. Res. 26, 2333-2355. doi: 10.1177/0962280215597579

Burgess, S., and Thompson, S. G. (2017). Interpreting findings from Mendelian randomization using the MR-Egger method. Eur. J. Epidemiol. 32, 377-389. doi: 10.1007/s10654-017-0255-x

Chrousos, G. P. (2009). Stress and disorders of the stress system. Nat. Rev. Endocrinol. 5, 374-381. doi: 10.1038/nrendo.2009.106

Cortese, S., Moreira-Maia, C. R., St Fleur, D., Morcillo-Penalver, C., Rohde, L. A., and Faraone, S. V. (2016). Association between ADHD and obesity: a systematic review and meta-analysis. Am. J. Psychiatry 173, 34-43. doi: 10.1176/appi.ajp. 2015.15020266

Coryell, W. H., Butcher, B. D., Burns, T. L., Dindo, L. N., Schlechte, J. A., and Calarge, C. A. (2016). Fat distribution and major depressive disorder in late adolescence. J. Clin. Psychiatry 77, 84-89. doi: 10.4088/JCP.14m0 9169

Crawford, K., Bracher-Smith, M., Owen, D., Kendall, K. M., Rees, E., Pardinas, A. F., et al. (2019). Medical consequences of pathogenic CNVs in adults: analysis of the UK Biobank. J. Med. Genet. 56, 131-138. doi: 10.1136/jmedgenet-2018105477

Davey Smith, G., and Ebrahim, S. (2005). What can mendelian randomisation tell us about modifiable behavioural and environmental exposures. BMJ 330, 1076-1079. doi: 10.1136/bmj.330.7499.1076

Davies, G., Marioni, R. E., Liewald, D. C., Hill, W. D., Hagenaars, S. P., Harris, S. E., et al. (2016). Genome-wide association study of cognitive functions and educational attainment in UK Biobank $(\mathrm{N}=112$ 151). Mol. Psychiatry 21, 758-767. doi: 10.1038/mp.2016.45

de Moor, M. H., Costa, P. T., Terracciano, A., Krueger, R. F., de Geus, E. J., Toshiko, T., et al. (2012). Meta-analysis of genome-wide association studies for personality. Mol. Psychiatry 17, 337-349. doi: 10.1038/mp.2010.128

Deary, V. (2018). Genetic contributions to self-reported tiredness. Mol. Psychiatry 23, 609-620. doi: 10.1038/mp.2017.5

Del Greco, M. F., Minelli, C., Sheehan, N. A., and Thomson, J. R. (2015). Detecting pleiotropy in Mendelian randomisation studies withsummary data and a continuous outcome. Stat. Med. 34, 2926-2940. doi: 10.1002/sim.6522

Demontis, D., Walters, R. K., Martin, J., Mattheisen, M., Als, T. D., Agerbo, E., et al. (2018). Discovery of the first genome-wide significant risk loci for attention deficit/hyperactivity disorder. Nat. Genet. 51, 63-75. doi: 10.1038/s41588-0180269-7

Dennis, E. L., Jahanshad, N., Braskie, M. N., Warstadt, N. M., Hibar, D. P., Kohannim, O., et al. (2014). Obesity gene NEGR1 associated with white matter integrity in healthy young adults. Neuroimage 102, 548-557. doi: 10.1016/j. neuroimage.2014.07.041

Després, J.-P. (2006). Abdominal obesity: the most prevalent cause of the metabolic syndrome and related cardiometabolic risk. Eur. Heart J. Suppl. 8(Suppl._B), B4-B12. doi: 10.1093/eurheartj/sul002

Di Paolo, T. (1994). Modulation of brain dopamine transmission by sex steroids. Rev. Neurosci. 5, 27-41.

Duncan, L., Yilmaz, Z., Gaspar, H., Walters, R., Goldstein, J., Anttila, V., et al. (2017). Significant locus and metabolic genetic correlations revealed in genomewide association study of anorexia Nervosa. Am. J. Psychiatry 174, 850-858. doi: 10.1176/appi.ajp.2017.16121402

Egger, J. I., Verhoeven, W. M., Verbeeck, W., and de Leeuw, N. (2014). Neuropsychological phenotype of a patient with a de novo $970 \mathrm{~kb}$ interstitial deletion in the distal 16p11.2 region. Neuropsychiatr. Dis. Treat. 10, 513-517. doi: 10.2147/NDT.S58684

Fernandez, B. A., Roberts, W., Chung, B., Weksberg, R., Meyn, S., Szatmari, P., et al. (2010). Phenotypic spectrum associated with de novo and inherited deletions and duplications at 16p11.2 in individuals ascertained for diagnosis of autism spectrum disorder. J. Med. Genet. 47, 195-203. doi: 10.1136/jmg.2009.06 9369

Frau, R., Bini, V., Pes, R., Pillolla, G., Saba, P., Devoto, P., et al. (2014). Inhibition of 17alpha-hydroxylase/C17,20 lyase reduces gating deficits consequent to dopaminergic activation. Psychoneuroendocrinology 39, 204-213. doi: 10.1016/ j.psyneuen.2013.09.014
Geiker, N. R. W., Astrup, A., Hjorth, M. F., Sjödin, A., Pijls, L., and Markus, C. R. (2018). Does stress influence sleep patterns, food intake, weight gain, abdominal obesity and weight loss interventions and vice versa? Obes. Rev. 19, 81-97. doi: 10.1111/obr.12603

Giuranna, J., Volckmar, A. L., Heinen, A., Peters, T., Schmidt, B., Spieker, A., et al. (2018). The effect of SH2B1 variants on expression of leptin- and insulininduced pathways in murine hypothalamus. Obes. Facts 11, 93-108. doi: 10. $1159 / 000486962$

Godar, S. C., and Bortolato, M. (2014). Gene-sex interactions in schizophrenia: focus on dopamine neurotransmission. Front. Behav. Neurosci. 8:71. doi: 10. 3389/fnbeh.2014.00071

Graff, M., Scott, R. A., Justice, A. E., Young, K. L., Feitosa, M. F., Barata, L., et al. (2017). Genome-wide physical activity interactions in adiposity - A metaanalysis of 200,452 adults. PLoS Genet. 13:e1006528. doi: 10.1371/journal.pgen. 1006528

Grove, J., Ripke, S., Als, T. D., Mattheisen, M., Walters, R., Won, H., et al. (2017). Common risk variants identified in autism spectrum disorder. bioRxiv [Preprint]. doi: 10.1101/224774

Consortium, G.T., Laboratory, D.A., Coordinating Center -Analysis Working, G., Statistical Methods groups-Analysis Working, G., Enhancing, G.g., Fund, N.I.H.C., et al. (2017). Genetic effects on gene expression across human tissues. Nature 550, 204-213. doi: 10.1038/nature24277

Gurpegui, M., Martinez-Ortega, J. M., Gutierrez-Rojas, L., Rivero, J., Rojas, C., and Jurado, D. (2012). Overweight and obesity in patients with bipolar disorder or schizophrenia compared with a non-psychiatric sample. Prog. Neuropsychopharmacol. Biol. Psychiatry 37, 169-175. doi: 10.1016/j.pnpbp. 2012.01.014

Halaris, A. (2019). Inflammation and depression but where does the inflammation come from? Curr. Opin. Psychiatry 32:1. doi: 10.1097/YCO.0000000000000531

Haycock, P. C., Burgess, S., Wade, K. H., Bowden, J., Relton, C., and Davey Smith, G. (2016). Best (but oft-forgotten) practices: the design, analysis, and interpretation of Mendelian randomization studies. Am. J. Clin. Nutr. 103, 965-978. doi: 10.3945/ajcn.115.118216

Heard-Costa, N. L., Zillikens, M. C., Monda, K. L., Johansson, A., Harris, T. B., Fu, M., et al. (2009). NRXN3 is a novel locus for waist circumference: a genome-wide association study from the CHARGE Consortium. PLoS Genet. 5:e1000539. doi: 10.1371/journal.pgen.1000539

Hebebrand, J., Peters, T., Schijven, D., Hebebrand, M., Grasemann, C., Winkler, T. W., et al. (2018). The role of genetic variation of human metabolism for BMI, mental traits and mental disorders. Mol. Metab. 12, 1-11. doi: 10.1016/ j.molmet.2018.03.015

Hill, W. D., Marioni, R. E., Maghzian, O., Ritchie, S. J., Hagenaars, S. P., McIntosh, A. M., et al. (2018). A combined analysis of genetically correlated traits identifies 187 loci and a role for neurogenesis and myelination in intelligence. Mol. Psychiatry 24, 169-181. doi: 10.1038/s41380-017-0001-5

Hinney, A., Volckmar, A. L., and Knoll, N. (2013). Melanocortin-4 receptor in energy homeostasis and obesity pathogenesis. Prog. Mol. Biol. Transl. Sci. 114 147-191. doi: 10.1016/B978-0-12-386933-3.00005-4

Jacquemont, S., Reymond, A., Zufferey, F., Harewood, L., Walters, R. G., Kutalik, Z., et al. (2011). Mirror extreme BMI phenotypes associated with gene dosage at the chromosome 16p11.2 locus. Nature 478, 97-102. doi: 10.1038/nature1 0406

Joo, Y., Kim, H., Lee, S., and Lee, S. (2019). Neuronal growth regulator 1-deficient mice show increased adiposity and decreased muscle mass. Int. J. Obes. 43, 1769-1782. doi: 10.1038/s41366-019-0376-2

Karasik, D., Zillikens, M. C., Hsu, Y. H., Aghdassi, A., Akesson, K., Amin, N., et al. (2019). Disentangling the genetics of lean mass. Am. J. Clin. Nutr. 109, 276-287. doi: 10.1093/ajen/nqy272

Karis, K., Eskla, K. L., Kaare, M., Taht, K., Tuusov, J., Visnapuu, T., et al. (2018). Altered expression profile of IgLON family of neural cell adhesion molecules in the dorsolateral prefrontal cortex of schizophrenic patients. Front. Mol. Neurosci. 11:8. doi: 10.3389/fnmol.2018.00008

Katikireddi, S. V., Green, M. J., Taylor, A. E., Davey Smith, G., and Munafo, M. R. (2018). Assessing causal relationships using genetic proxies for exposures: an introduction to Mendelian randomization. Addiction 113, 764-774. doi: 10. 1111/add.14038

Kaur, Y., Wang, D. X., Liu, H. Y., and Meyre, D. (2018). Comprehensive identification of pleiotropic loci for body fat distribution using the NHGRI-EBI 
Catalog of published genome-wide association studies. Obes. Rev. 20, 385-406. doi: $10.1111 /$ obr.12806

Kim, J. H., Kim, J. H., Park, P. W., Machann, J., Roden, M., Lee, S. W., et al. (2017). Body and liver fat content and adipokines in schizophrenia: a magnetic resonance imaging and spectroscopy study. Psychopharmacology 234, 19231932. doi: 10.1007/s00213-017-4598-5

Kim, S. H., Moon, J. Y., Sasano, H., Choi, M. H., and Park, M. J. (2016). Body fat mass is associated with ratio of steroid metabolites reflecting 17,20-lyase activity in prepubertal girls. J. Clin. Endocrinol. Metab. 101, 4653-4660. doi: 10.1210/jc.2016-2515

Kmetova Sivonova, M., Jurecekova, J., Tatarkova, Z., Kaplan, P., Lichardusova, L., and Hatok, J. (2017). The role of CYP17A1 in prostate cancer development: structure, function, mechanism of action, genetic variations and its inhibition. Gen. Physiol. Biophys. 36, 487-499. doi: 10.4149/gpb_201 7024

Konarzewska, B., Stefanska, E., Wendolowicz, A., Cwalina, U., Golonko, A., Malus, A., et al. (2014). Visceral obesity in normal-weight patients suffering from chronic schizophrenia. BMC Psychiatry 14:35. doi: 10.1186/1471-244X-14-35

König, I. R., and Greco, F. M. D. (2018). Mendelian randomization: progressing towards understanding causality. Ann. Neurol. 84, 176-177. doi: 10.1002/ana. 25293

Lasselin, J., Elsenbruch, S., Lekander, M., Axelsson, J., Karshikoff, B., Grigoleit, J. S., et al. (2016). Mood disturbance during experimental endotoxemia: predictors of state anxiety as a psychological component of sickness behavior. Brain Behav. Immun. 57, 30-37. doi: 10.1016/j.bbi.2016.01.003

LeBlanc, M., Zuber, V., Thompson, W. K., Andreassen, O. A., Schizophrenia and Bipolar Disorder Working Groups of the Psychiatric Genomics Consortium, Frigessi, A., et al. (2018). A correction for sample overlap in genomewide association studies in a polygenic pleiotropy-informed framework. BMC Genomics 19:494. doi: 10.1186/s12864-018-4859-7

Lee, J. J., Wedow, R., Okbay, A., Kong, E., Maghzian, O., Zacher, M., et al. (2018). Gene discovery and polygenic prediction from a genome-wide association study of educational attainment in 1.1 million individuals. Nat. Genet. 50, 1112-1121. doi: 10.1038/s41588-018-0147-3

Lee, S. E., West, KP Jr, Cole, R. N., Schulze, K. J., Wu, L. S., Yager, J. D., et al. (2016). General intelligence is associated with subclinical inflammation in Nepalese children: a population-based plasma proteomics study. Brain Behav Immun 56, 253-263. doi: 10.1016/j.bbi.2016.03.023

Leslie, R., O'Donnell, C. J., and Johnson, A. D. (2014). GRASP: analysis of genotype-phenotype results from 1390 genome-wide association studies and corresponding open access database. Bioinformatics 30, i185-i194. doi: 10.1093/ bioinformatics/btu273

Levinson, D. F., Mostafavi, S., Milaneschi, Y., Rivera, M., Ripke, S., Wray, N. R., et al. (2014). Genetic studies of major depressive disorder: Why are there no genome-wide association study findings and what can we do about it? Biol. Psychiatry 76, 510-512. doi: 10.1016/j.biopsych.2014. 07.029

Levy, D., Ehret, G. B., Rice, K., Verwoert, G. C., Launer, L. J., Dehghan, A., et al. (2009). Genome-wide association study of blood pressure and hypertension. Nat. Genet. 41, 677-687. doi: 10.1038/ng.384

Li, Z., Chang, S. H., Zhang, L. Y., Gao, L., and Wang, J. (2014). Molecular genetic studies of ADHD and its candidate genes: a review. Psychiatry Res. 219, 10-24. doi: 10.1016/j.psychres.2014.05.005

Lim, B. K., Huang, K. W., Grueter, B. A., Rothwell, P. E., and Malenka, R. C. (2012). Anhedonia requires MC4R-mediated synaptic adaptations in nucleus accumbens. Nature 487, 183-189. doi: 10.1038/nature11160

Liu, J., Garza, J. C., Li, W., and Lu, X. Y. (2013a). Melanocortin-4 receptor in the medial amygdala regulates emotional stress-induced anxiety-like behaviour, anorexia and corticosterone secretion. Int. J. Neuropsychopharmacol. 16, 105120. doi: $10.1017 /$ S146114571100174X

Liu, M. J., Bao, S., Galvez-Peralta, M., Pyle, C. J., Rudawsky, A. C., Pavlovicz, R. E., et al. (2013b). ZIP8 regulates host defense through zinc-mediated inhibition of NF-kappaB. Cell Rep. 3, 386-400. doi: 10.1016/j.celrep.2013.01.009

Lo, M. T., Hinds, D. A., Tung, J. Y., Franz, C., Fan, C. C., Wang, Y., et al. (2017). Genome-wide analyses for personality traits identify six genomic loci and show correlations with psychiatric disorders. Nat. Genet. 49, 152-156. doi: $10.1038 /$ ng. 3736
Locke, A. E., Kahali, B., Berndt, S. I., Justice, A. E., Pers, T. H., Day, F. R., et al. (2015). Genetic studies of body mass index yield new insights for obesity biology. Nature 518, 197-206. doi: 10.1038/nature14177

Loja-Chango, R., Pérez-López, F. R., Simoncini, T., Escobar, G. S., and Chedraui, P. (2016). Increased mood symptoms in postmenopausal women related to the polymorphism rs743572 of the CYP17 Al gene. Gynecol. Endocrinol. 32, 827-830. doi: 10.1080/09513590.2016.1177015

Loos, R. J., Lindgren, C. M., Li, S., Wheeler, E., Zhao, J. H., Prokopenko, I., et al. (2008). Common variants near MC4R are associated with fat mass, weight and risk of obesity. Nat. Genet. 40, 768-775. doi: 10.1038/ng.140

Lu, Y., Day, F. R., Gustafsson, S., Buchkovich, M. L., Na, J., Bataille, V., et al. (2016). New loci for body fat percentage reveal link between adiposity and cardiometabolic disease risk. Nat. Commun. 7:10495. doi: 10.1038/ ncomms 10495

Luciano, M., Hagenaars, S. P., Davies, G., Hill, W. D., Clarke, T.-K., Shirali, M., et al. (2018). Association analysis in over 329,000 individuals identifies 116 independent variants influencing neuroticism. Nat. Genet. 50, 6-11. doi: 10. 1038/s41588-017-0013-8

Luppino, F. S., Bouvy, P. F., Giltay, E. J., Penninx, B. W., and Zitman, F. G. (2014). The metabolic syndrome and related characteristics in major depression: inpatients and outpatients compared: metabolic differences across treatment settings. Gen. Hosp. Psychiatry 36, 509-515. doi: 10.1016/j.genhosppsych.2014. 05.018

Luppino, F. S., de Wit, L. M., Bouvy, P. F., Stijnen, T., Cuijpers, P., Penninx, B. W., et al. (2010). Overweight, obesity, and depression: a systematic review and meta-analysis of longitudinal studies. Arch. Gen. Psychiatry 67, 220-229. doi: 10.1001/archgenpsychiatry.2010.2

Machiela, M. J., and Chanock, S. J. (2015). LDlink: a web-based application for exploring population-specific haplotype structure and linking correlated alleles of possible functional variants. Bioinformatics 31, 3555-3557. doi: 10.1093/ bioinformatics/btv402

Maillard, A. M., Hippolyte, L., Rodriguez-Herreros, B., Chawner, S. J. R. A., Dremmel, D., Agüera, Z., et al. (2016). 16p11.2 Locus modulates response to satiety before the onset of obesity. Int. J. Obes. 40, 870-876. doi: 10.1038/ijo. 2015.247

Martin-Brevet, S., Rodriguez-Herreros, B., Nielsen, J. A., Moreau, C., Modenato, C., Maillard, A. M., et al. (2018). Quantifying the effects of 16p11.2 copy number variants on brain structure: a multisite genetic-first study. Biol. Psychiatry 84, 253-264. doi: 10.1016/j.biopsych.2018.02.1176

McCarthy, S. E., Makarov, V., Kirov, G., Addington, A. M., McClellan, J., Yoon, S., et al. (2009). Microduplications of 16 p11.2 are associated with schizophrenia. Nat. Genet. 41, 1223-1227. doi: 10.1038/ng.474

Meyer-Lindenberg, A., and Tost, H. (2012). Neural mechanisms of social risk for psychiatric disorders. Nat. Neurosci. 15, 663-668. doi: 10.1038/nn.3083

Milaneschi, Y., Simmons, W. K., van Rossum, E. F. C., and Penninx, B. W. (2019). Depression and obesity: evidence of shared biological mechanisms. Mol. Psychiatry 24, 18-33. doi: 10.1038/s41380-018-0017-5

Monda, K. L., Chen, G. K., Taylor, K. C., Palmer, C., Edwards, T. L., Lange, L. A., et al. (2013). A meta-analysis identifies new loci associated with body mass index in individuals of African ancestry. Nat. Genet. 45, 690-696. doi: $10.1038 /$ ng. 2608

Morris, J., Bailey, M. E. S., Baldassarre, D., Cullen, B., de Faire, U., Ferguson, A., et al. (2019). Genetic variation in CADM2 as a link between psychological traits and obesity. Sci. Rep. 9:7339. doi: 10.1038/s41598-019-43861-9

Mühlig, Y., Antel, J., Föcker, M., and Hebebrand, J. (2015). Are bidirectional associations of obesity and depression already apparent in childhood and adolescence as based on high-quality studies? A systematic review. Obes. Rev. 17, 235-249. doi: 10.1111/obr.12357

Muller, N. (2019). COX-2 inhibitors, aspirin, and other potential antiinflammatory treatments for psychiatric disorders. Front. Psychiatry 10:375. doi: $10.3389 /$ fpsyt. 2019.00375

Mullins, N., and Lewis, C. M. (2017). Genetics of depression: progress at last. Curr. Psychiatry Rep. 19:43. doi: 10.1007/s11920-017-0803-9

Murakami, I., Oh, Y., Morimoto, A., Sano, H., Kanzaki, S., Matsushita, M., et al. (2015). Acute-phase ITIH4 levels distinguish multi-system from single-system Langerhans cell histiocytosis via plasma peptidomics. Clin. Proteomics 12:16. doi: 10.1186/s12014-015-9089-2 
Niarchou, M., Chawner, S., Doherty, J. L., Maillard, A. M., Jacquemont, S., Chung, W. K., et al. (2019). Psychiatric disorders in children with 16p11.2 deletion and duplication. Transl. Psychiatry 9:8. doi: 10.1038/s41398-018-0339-8

Nigg, J. T., Johnstone, J. M., Musser, E. D., Long, H. G., Willoughby, M. T., and Shannon, J. (2016). Attention-deficit/hyperactivity disorder (ADHD) and being overweight/obesity: new data and meta-analysis. Clin. Psychol. Rev. 43, 67-79. doi: 10.1016/j.cpr.2015.11.005

Nikpay, M., Seda, O., Tremblay, J., Petrovich, M., Gaudet, D., Kotchen, T. A., et al. (2012). Genetic mapping of habitual substance use, obesity-related traits, responses to mental and physical stress, and heart rate and blood pressure measurements reveals shared genes that are overrepresented in the neural synapse. Hypertens. Res. 35, 585-591. doi: 10.1038/hr.2011.233

Nothen, M. M., Degenhardt, F., and Forstner, A. J. (2019). [Breakthrough in understanding the molecular causes of psychiatric disorders]. Nervenarzt 90 , 99-106. doi: 10.1007/s00115-018-0670-6

Okada, Y., Kubo, M., Ohmiya, H., Takahashi, A., Kumasaka, N., Hosono, N., et al. (2012). Common variants at CDKAL1 and KLF9 are associated with body mass index in east Asian populations. Nat. Genet. 44, 302-306. doi: 10.1038/ng.1086

Okbay, A. (2016a). Genetic variants associated with subjective well-being, depressive symptoms, and neuroticism identified through genome-wide analyses. Nat. Genet. 48, 624-633. doi: 10.1038/ng.3552

Okbay, A. (2016b). Genome-wide association study identifies 74 loci associated with educational attainment. Nature 533, 539-542. doi: 10.1038/nature17671

Otowa, T., Hek, K., Lee, M., Byrne, E. M., Mirza, S. S., Nivard, M. G., et al. (2016). Meta-analysis of genome-wide association studies of anxiety disorders. Mol. Psychiatry 21, 1391-1399. doi: 10.1038/mp.2015.197

Pardinas, A. F., Holmans, P., Pocklington, A. J., Escott-Price, V., Ripke, S., Carrera, N., et al. (2018). Common schizophrenia alleles are enriched in mutationintolerant genes and in regions under strong background selection. Nat. Genet. 50, 381-389. doi: 10.1038/s41588-018-0059-2

Patalay, P., and Hardman, C. A. (2019). Comorbidity, codevelopment, and temporal associations between body mass index and internalizing symptoms from early childhood to adolescence. JAMA Psychiatry 76, 721-729. doi: 10. 1001/jamapsychiatry.2019.0169

Pellissier, F., Gerber, A., Bauer, C., Ballivet, M., and Ossipow, V. (2007). The adhesion molecule Necl-3/SynCAM-2 localizes to myelinated axons, binds to oligodendrocytes and promotes cell adhesion. BMC Neurosci. 8:90. doi: 10. 1186/1471-2202-8-90

Peng, Z., Xueb, G., Chen, W., and Xia, S. (2019). Environmental inhibitors of the expression of cytochrome P450 17A1 in mammals. Environ. Toxicol. Pharmacol. 69, 16-25. doi: 10.1016/j.etap.2019.02.007

Perry, B. I., Upthegrove, R., Thompson, A., Marwaha, S., Zammit, S., Singh, S. P., et al. (2019). Dysglycaemia, inflammation and psychosis: findings from the UK ALSPAC Birth Cohort. Schizophr. Bull. 45, 330-338. doi: 10.1093/schbul/ sby040

Pettersson, E., Lichtenstein, P., Larsson, H., Song, J., Attention Deficit/Hyperactivity Disorder Working Group of the iPsych-Broad-Pgc Consortium, Autism Spectrum Disorder Working Group of the iPSYCHBroad-Pgc Consortium, et al. (2019). Genetic influences on eight psychiatric disorders based on family data of 4408646 full and half-siblings, and genetic data of 333748 cases and controls. Psychol. Med. 49, 1166-1173. doi: $10.1017 /$ S0033291718002039

Pflanz, S., Timans, J. C., Cheung, J., Rosales, R., Kanzler, H., Gilbert, J., et al. (2002). IL-27, a Heterodimeric Cytokine Composed of EBI3 and p28 Protein, Induces Proliferation of Naive CD4+T Cells. Immunity 16, 779-790. doi: 10. 1016/S1074-7613(02)00324-2

Pischedda, F., and Piccoli, G. (2015). The IgLON family member Negrl promotes neuronal arborization acting as soluble factor via FGFR2. Front. Mol. Neurosci. 8:89. doi: $10.3389 /$ fnmol.2015.00089

Pulit, S. L., Stoneman, C., Morris, A. P., Wood, A. R., Glastonbury, C. A., Tyrrell, J., et al. (2019). Meta-analysis of genome-wide association studies for body fat distribution in 694649 individuals of European ancestry. Hum. Mol. Genet. 28, 166-174. doi: 10.1093/hmg/ddy327

Qi, X.-R., Luchetti, S., Verwer, R. W. H., Sluiter, A. A., Mason, M. R. J., Zhou, J.N., et al. (2018). Alterations in the steroid biosynthetic pathways in the human prefrontal cortex in mood disorders: a post-mortem study. Brain Pathol. 28, 536-547. doi: 10.1111/bpa.12548
Qureshi, A. Y., Mueller, S., Snyder, A. Z., Mukherjee, P., Berman, J. I., Roberts, T. P., et al. (2014). Opposing brain differences in 16p11.2 deletion and duplication carriers. J. Neurosci. 34, 11199-11211. doi: 10.1523/JNEUROSCI.1366-14.2014

Rask-Andersen, M., Karlsson, T., Ek, W. E., and Johansson, A. (2019). Genomewide association study of body fat distribution identifies adiposity loci and sex-specific genetic effects. Nat. Commun. 10:339. doi: 10.1038/s41467-01808000-4

Rathjen, T., Yan, X., Kononenko, N. L., Ku, M. C., Song, K., Ferrarese, L., et al. (2017). Regulation of body weight and energy homeostasis by neuronal cell adhesion molecule 1. Nat. Neurosci. 20, 1096-1103. doi: 10.1038/nn.4590

Richardson, L. P., Davis, R., Poulton, R., McCauley, E., Moffitt, T. E., Caspi, A., et al. (2003). A longitudinal evaluation of adolescent depression and adult obesity. Arch. Pediatr. Adolesc. Med. 157, 739-745. doi: 10.1001/archpedi.157.8.739

Richardson, T. G., Harrison, S., Hemani, G., and Davey Smith, G. (2019a). An atlas of polygenic risk score associations to highlight putative causal relationships across the human phenome. eLife 8:e43657. doi: 10.7554/eLife.43657

Richardson, T. G., Harrison, S., Hemani, G., and Davey Smith, G. (2019b). Data From: An Atlas of Polygenic Risk Score Associations to Highlight Putative Causal Relationships Across the Human Phenome. Available online at: http://dx.doi.org/ 10.5061/dryad.h18c66b (accessed May 21, 2019).

Ripke, S. (2014). Biological insights from 108 schizophrenia-associated genetic loci. Nature 511, 421-427. doi: 10.1038/nature13595

Sabban, E. L., Serova, L. I., Alaluf, L. G., Laukova, M., and Peddu, C. (2015). Comparative effects of intranasal neuropeptide $\mathrm{Y}$ and HS014 in preventing anxiety and depressive-like behavior elicited by single prolonged stress. Behav. Brain Res. 295, 9-16. doi: 10.1016/j.bbr.2014.12.038

Sanchez, M. G., Bourque, M., Morissette, M., and Di Paolo, T. (2010). Steroidsdopamine interactions in the pathophysiology and treatment of CNS disorders. CNS Neurosci. Ther. 16, e43-e71. doi: 10.1111/j.1755-5949.2010.00163.x

Schneider, H. J. (2010). The predictive value of different measures of obesity for incident cardiovascular events and mortality. J. Clin. Endocrinol. Metab. 95, 1777-1785. doi: 10.1210/jc.2009-1584

Seeman, M. V. (1996). The role of estrogen in schizophrenia. J. Psychiatry Neurosci. 21, 123-127.

Shah, P., Iwata, Y., Caravaggio, F., Plitman, E., Brown, E. E., Kim, J., et al. (2019). Alterations in body mass index and waist-to-hip ratio in never and minimally treated patients with psychosis: a systematic review and metaanalysis. Schizophr. Res. 208, 420-429. doi: 10.1016/j.schres.2019.01.005

Sharma, A. (2016). Systems genomics support for immune and inflammation hypothesis of depression. Curr. Neuropharmacol. 14, 749-758. doi: 10.2174/ $1570159 \times 14666160106155331$

Shungin, D. (2015). New genetic loci link adipose and insulin biology to body fat distribution. Nature 518, 187-196. doi: 10.1038/nature14132

Sklar, P. (2011). Large-scale genome-wide association analysis of bipolar disorder identifies a new susceptibility locus near ODZ4. Nat. Genet. 43, 977-983. doi: 10.1038/ng.943

Sniekers, S., Stringer, S., Watanabe, K., Jansen, P. R., Coleman, J. R. I., Krapohl, E., et al. (2017). Genome-wide association meta-analysis of 78,308 individuals identifies new loci and genes influencing human intelligence. Nat. Genet. 49, 1107-1112. doi: $10.1038 / \mathrm{ng} .3869$

Snijder, M. B., van Dam, R. M., Visser, M., and Seidell, J. C. (2006). What aspects of body fat are particularly hazardous and how do we measure them? Int. J. Epidemiol. 35, 83-92. doi: 10.1093/ije/dyi253

Sonderby, I. E., Gustafsson, O., Doan, N. T., Hibar, D. P., Martin-Brevet, S., Abdellaoui, A., et al. (2018). Dose response of the 16p11.2 distal copy number variant on intracranial volume and basal ganglia. Mol. Psychiatry 25, 584-602. doi: 10.1038/s41380-018-0118-1

Stahl, E., Forstner, A., McQuillin, A., Ripke, S., Ophoff, R., Scott, L., et al. (2017). Genomewide association study identifies 30 loci associated with bipolar disorder. bioRxiv [Preprint]. doi: 10.1101/173062

Stefansson, H., Meyer-Lindenberg, A., Steinberg, S., Magnusdottir, B., Morgen, K., Arnarsdottir, S., et al. (2014). CNVs conferring risk of autism or schizophrenia affect cognition in controls. Nature 505, 361-366. doi: 10.1038/nature12818

Strassnig, M., Clarke, J., Mann, S., Remington, G., and Ganguli, R. (2017). Body composition, pre-diabetes and cardiovascular disease risk in early schizophrenia. Early Interv. Psychiatry 11, 229-236. doi: 10.1111/eip. 12225 
Sugawara, N., Yasui-Furukori, N., Tsuchimine, S., Fujii, A., Sato, Y., Saito, M., et al. (2012). Body composition in patients with schizophrenia: Comparison with healthy controls. Ann. Gen. Psychiatry 11:11. doi: 10.1186/1744-859X-11-11

The UniProt Consortium, (2017). UniProt: the universal protein knowledgebase. Nucleic Acids Res. 45, D158-D169. doi: 10.1093/nar/gkw1099

van der Valk, E. S., Savas, M., and van Rossum, E. F. C. (2018). Stress and obesity: Are there more susceptible individuals? Curr. Obes. Rep. 7, 193-203. doi: 10.1007/s13679-018-0306-y

van Vliet-Ostaptchouk, J. V., den Hoed, M., Luan, J., Zhao, J. H., Ong, K. K., van der Most, P. J., et al. (2013). Pleiotropic effects of obesity-susceptibility loci on metabolic traits: a meta-analysis of up to 37,874 individuals. Diabetologia 56, 2134-2146. doi: 10.1007/s00125-013-2985-y

Van Woudenberg, M., Shin, J., Bernard, M., Syme, C., Abrahamowicz, M., Leonard, G., et al. (2015). CYP17A1 and blood pressure reactivity to stress in adolescence. Int. J. Hypertens. 2015:734586. doi: 10.1155/2015/734586

Volckmar, A. L., Bolze, F., Jarick, I., Knoll, N., Scherag, A., Reinehr, T., et al. (2012). Mutation screen in the GWAS derived obesity gene SH2B1 including functional analyses of detected variants. BMC Med. Genomics 5:65. doi: 10.1186/17558794-5-65

Volckmar, A. L., Song, J. Y., Jarick, I., Putter, C., Gobel, M., Horn, L., et al. (2015). Fine mapping of a GWAS-derived obesity candidate region on chromosome 16p11.2. PLoS One 10:e0125660. doi: 10.1371/journal.pone.0125660

Walters, R. G., Jacquemont, S., Valsesia, A., de Smith, A. J., Martinet, D., Andersson, J., et al. (2010). A new highly penetrant form of obesity due to deletions on chromosome 16p11.2. Nature 463, 671-675. doi: 10.1038/nature08727

Wang, D., Ma, J., Zhang, S., Hinney, A., Hebebrand, J., Wang, Y., et al. (2010). Association of the MC4R V103I polymorphism with obesity: a Chinese casecontrol study and meta-analysis in 55,195 individuals. Obesity 18, 573-579. doi: $10.1038 /$ oby.2009.268

Welter, D., MacArthur, J., Morales, J., Burdett, T., Hall, P., Junkins, H., et al. (2014). The NHGRI GWAS Catalog, a curated resource of SNP-trait associations. Nucleic Acids Res. 42, D1001-D1006. doi: 10.1093/nar/gkt1229

Wen, W., Zheng, W., Okada, Y., Takeuchi, F., Tabara, Y., Hwang, J.-Y., et al. (2014). Meta-analysis of genome-wide association studies in East Asianancestry populations identifies four new loci for body mass index. Hum. Mol. Genet. 23, 5492-5504. doi: 10.1093/hmg/ddu248

Willer, C. J. (2009). Six new loci associated with body mass index highlight a neuronal influence on body weight regulation. Nat. Genet. 41, 25-34. doi: 10. 1038/ng.287

Winkler, T. W., Justice, A. E., Graff, M., Barata, L., Feitosa, M. F., Chu, S., et al. (2015). The influence of age and sex on genetic associations with adult body size and shape: a large-scale genome-wide interaction study. PLoS Genet. 11:e1005378. doi: 10.1371/journal.pgen.1005378

Wray, N. R., Ripke, S., Mattheisen, M., Trzaskowski, M., Byrne, E. M., Abdellaoui, A., et al. (2018). Genome-wide association analyses identify 44 risk variants and refine the genetic architecture of major depression. Nat. Genet. 50, 668-681. doi: 10.1038/s41588-018-0090-3

Xu, P., Grueter, B. A., Britt, J. K., McDaniel, L., Huntington, P. J., Hodge, R., et al. (2013). Double deletion of melanocortin 4 receptors and SAPAP3 corrects compulsive behavior and obesity in mice. Proc. Natl. Acad. Sci. U.S.A. 110, 10759-10764. doi: 10.1073/pnas.13081 95110

Yan, X., Wang, Z., Schmidt, V., Gauert, A., Willnow, T. E., Heinig, M., et al. (2018). Cadm2 regulates body weight and energy homeostasis in mice. Mol. Metab. 8, 180-188. doi: 10.1016/j.molmet.2017.11.010

Yengo, L., Sidorenko, J., Kemper, K. E., Zheng, Z., Wood, A. R., Weedon, M. N., et al. (2018). Meta-analysis of genome-wide association studies for height and body mass index in approximately 700000 individuals of European ancestry. Hum. Mol. Genet. 27, 3641-3649. doi: 10.1093/hmg/ ddy 271

Yilmaz, Z., Davis, C., Loxton, N. J., Kaplan, A. S., Levitan, R. D., Carter, J. C., et al. (2015). Association between MC4R rs17782313 polymorphism and overeating behaviors. Int. J. Obes. 39, 114-120. doi: 10.1038/ijo.2014.79

Yoshimoto, F. K., and Auchus, R. J. (2015). The diverse chemistry of cytochrome P450 17A1 (P450c17. CYP17A1). J. Steroid Biochem. Mol. Biol. 151, 52-65. doi: 10.1016/j.jsbmb.2014.11.026

Zhao, Q., Wang, J., Hemani, G., Bowden, J., and Small, D. S. (2019). Statistical interference in two-sammple summary data Mendelian Randomization Robust Adjusted Profile Score. arXiv [Preprint]. Available online at: https://arxiv.org/ abs/1801.09652 (accessed January 28, 2020).

Zheng, J. (2017). LD Hub: a centralized database and web interface to perform LD score regression that maximizes the potential of summary level GWAS data for SNP heritability and genetic correlation analysis. Bioinformatics 33, 272-279. doi: 10.1093/bioinformatics/btw613

Zufferey, F., Sherr, E. H., Beckmann, N. D., Hanson, E., Maillard, A. M., Hippolyte, L., et al. (2012). A $600 \mathrm{~kb}$ deletion syndrome at $16 \mathrm{p} 11.2$ leads to energy imbalance and neuropsychiatric disorders. J. Med. Genet. 49, 660-668. doi: 10.1136/jmedgenet-2012-101203

Conflict of Interest: The authors declare that the research was conducted in the absence of any commercial or financial relationships that could be construed as a potential conflict of interest.

Copyright (c) 2020 Peters, Nüllig, Antel, Naaresh, Laabs, Tegeler, Amhaouach, Libuda, Hinney and Hebebrand. This is an open-access article distributed under the terms of the Creative Commons Attribution License (CC BY). The use, distribution or reproduction in other forums is permitted, provided the original author(s) and the copyright owner(s) are credited and that the original publication in this journal is cited, in accordance with accepted academic practice. No use, distribution or reproduction is permitted which does not comply with these terms. 